\title{
Morphometric structural diversity of a natural armor assembly investigated by 2D continuum strain analysis
}

\author{
Authors: Swati Varshney, ${ }^{\mathrm{a}}$ Juha Song, ${ }^{\mathrm{a}}$ Yaning Li, ${ }^{\mathrm{b}}$ Mary C. Boyce, ${ }^{\mathrm{c}}$ and Christine Ortiz ${ }^{\mathrm{a}}$ \\ ${ }^{a}$ Department of Materials Science and Engineering, Massachusetts Institute of Technology, 77 \\ Massachusetts Avenue, Cambridge, MA 02139, USA. \\ ${ }^{\mathrm{b}}$ Department of Mechanical Engineering, Massachusetts Institute of Technology, 77 Massachusetts \\ Avenue, Cambridge, MA 02139, USA. Present address: Department of Mechanical Engineering, \\ University of New Hampshire, Durham, NH 03824, USA \\ ${ }^{c}$ Department of Mechanical Engineering, Massachusetts Institute of Technology, 77 Massachusetts \\ Avenue, Cambridge, MA 02139, USA. Present address: School of Engineering and Applied Sciences, \\ Columbia University, New York, NY 10027, USA \\ * Corresponding author: \\ Professor Christine Ortiz \\ Massachusetts Institute of Technology \\ 77 Massachusetts Avenue, RM 3-132 \\ Cambridge, MA, 02139, USA. \\ Phone: $617-253-1957$ \\ Fax: 617-253-5620 \\ Email: cortiz@mit.edu
}




\section{Abstract}

Many armored fish scale assemblies use geometric heterogeneity of subunits as a design parameter to provide tailored biomechanical flexibility while maintaining protection from external penetrative threats. This study analyzes the spatially varying shape of individual ganoid scales as a structural element in a biological system, the exoskeleton of the armored fish P. senegalus (bichir). X-ray microcomputed tomography is used to generate digital 3D reconstructions of the mineralized scales. Landmark-based geometric morphometrics is used to measure the geometric variation among scales and to define a set of geometric parameters to describe shape variation. A formalism using continuum mechanical strain analysis is developed to quantify the spatial geometry change of the scales and illustrate the mechanisms of shape morphing between scales. Five scale geometry variants are defined (average, anterior, tail, ventral, and pectoral fin) and their functional implications are discussed in terms of the interscale mobility mechanisms that enable flexibility within the exoskeleton. The results suggest that shape variation in materials design, inspired by structural biological materials, can allow for tunable behavior in flexible composites made of segmented scale assemblies to achieve enhanced user mobility, custom fit, and flexibility around joints for a variety of protective applications.

Keywords: Morphometrics, geometric morphometrics, fish armor, flexible exoskeleton, bioinspiration 


\section{Introduction}

Structural biological materials integrate heterogeneity in classical material structure (e.g. materials selection, crystallography, local surface chemistry) with morphometry (geometry or shape-based design parameters) to tune their functionality to physiological need and organism survival (Arciszewski \& Cornell, 2006; Ortiz \& Boyce, 2008; Oyen et al., 2006; Wainwright et al., 1976; Chen et al., 2012; Meyers et al., 2008; Weiner \& Addadi, 1997; Dunlop et al., 2011). Armored fish have evolved protective exoskeletons that provide penetration resistance while maintaining flexibility through scale geometry, arrangement, and interlocking mechanisms (Song et al., 2010; Browning et al., 2013; Yang et al., 2013). One model species, Polypterus senegalus (bichir), possesses a mineralized, full-coverage exoskeleton with flexibility (Sire, 1990; Daget et al., 2001) for axial bendbarting and torsion (Gemballa \& Bartsch, 2002), escape maneuvers (Tytell \& Lauder, 2002), and recoil aspiration (Brainerd, 1994). Heterogeneity in material structure and properties provides protection: each mineralized scale has a quad-layered nanocomposite microstructure with a porous architecture for penetration resistance, toughness, and noncatastrophic pathways for energy dissipation while exhibiting load-dependent material properties, circumferential surface cracking, minimized weight, and tailored layer thickness for threat matching to predatory biting attacks (Bruet et al., 2008; Song et al., 2011; Wang et al., 2009; Han et al., 2011; Meunier, 1987; Meinke et al., 1979; Sire, 1994). Scales are assembled into overlapping columns with organic connective tissue reinforcing the articulating joints and attaching the scales to the dermis (Gemballa \& Bartsch, 2002; Pearson, 1981). Scale shape and size vary spatially, yet the integument achieves high bending curvatures in every location due to scale geometry and joint articulation (Gemballa \& Bartsch, 2002). Little is known regarding geometric heterogeneity's contribution to the local, interscale mobility mechanisms that enable global flexibility.

This study examines shape and shape variation as a structural design element in a biological system, the armored exoskeleton of P. senegalus. Scale geometry is analyzed along the anteroposterior and 
dorsoventral axes of the P. senegalus exoskeleton and into its pectoral fin. Digital 3D reconstructions of the mineralized scales are generated using X-ray microcomputed tomography $(\mu \mathrm{CT})$. Geometric variation among scales is investigated using landmark-based geometric morphometrics (GM), and a set of geometric parameters describing shape variation are defined. Furthermore, a continuum mechanical strain formalism is developed to quantify the morphometric variation. GM transformation grids and strain plots illustrate the mechanisms of shape morphing by modeling shape variation through the complex loading conditions of heterogeneous strain fields. Five scale geometry variants are defined, and their functional implications are discussed in terms of the interscale mobility mechanisms that allow flexibility within the exoskeleton. The method is further discussed to show that the application of strain fields to a given geometry can achieve a target morphology. While GM is often used to visualize inter-organismal geometric variation as a metric for species differentiation (Elewa, 2010), this intraspecies analysis probes morphometric heterogeneity of subunits within one specimen. The results suggest that the use of shape and shape variation as a materials design parameter can be used to tune the behavior of bioinspired, flexible composite materials for a protective applications.

\section{Materials and Methods}

\subsection{X-ray Microcomputed Tomography $(\mu \mathrm{CT})$}

Scales from a deceased $P$. senegalus specimen $(22 \mathrm{~cm}$ body length) were scanned by $\mu \mathrm{CT}$ (VivaCT40, Scanco Medical AG, Switzerland) operated at $45 \mathrm{kV}$ and $177 \mu \mathrm{A}$ with no filter on the incident x-rays following our previously published procedure (Song et al., 2010). Microtomographic slices were recorded every $8-18 \mu \mathrm{m}$ with $360^{\circ}$ rotation and were reconstructed with $8 \times 8 \mu \mathrm{m}$ to $18 \times 18 \mu \mathrm{m}$ volume elements (voxels) in plane. A constrained 3D Gaussian filter $(\sigma=0.8$ and support $=1$ ) was used to partially suppress noise in the volumes. Medical imaging software (MIMICS 15.1, Materialise, Belgium) was used to threshold the reconstructed transverse slices by white value with lower $(7,500)$ and upper $(30,000)$ values chosen manually through trial and error to isolate the mineralized scales from the organic 
connective tissue elements attached to the scales, and to build 3D polygonal meshes, using a bilinear and interplane interpolation algorithm, which were exported as stereo-lithography objects (STL). The specimen used in this study, shown in the $\mu \mathrm{CT}$ reconstruction in Fig. 1a, has 56 columns and 18 rows of scales identified subsequently by their column and row number (C\#R\#).
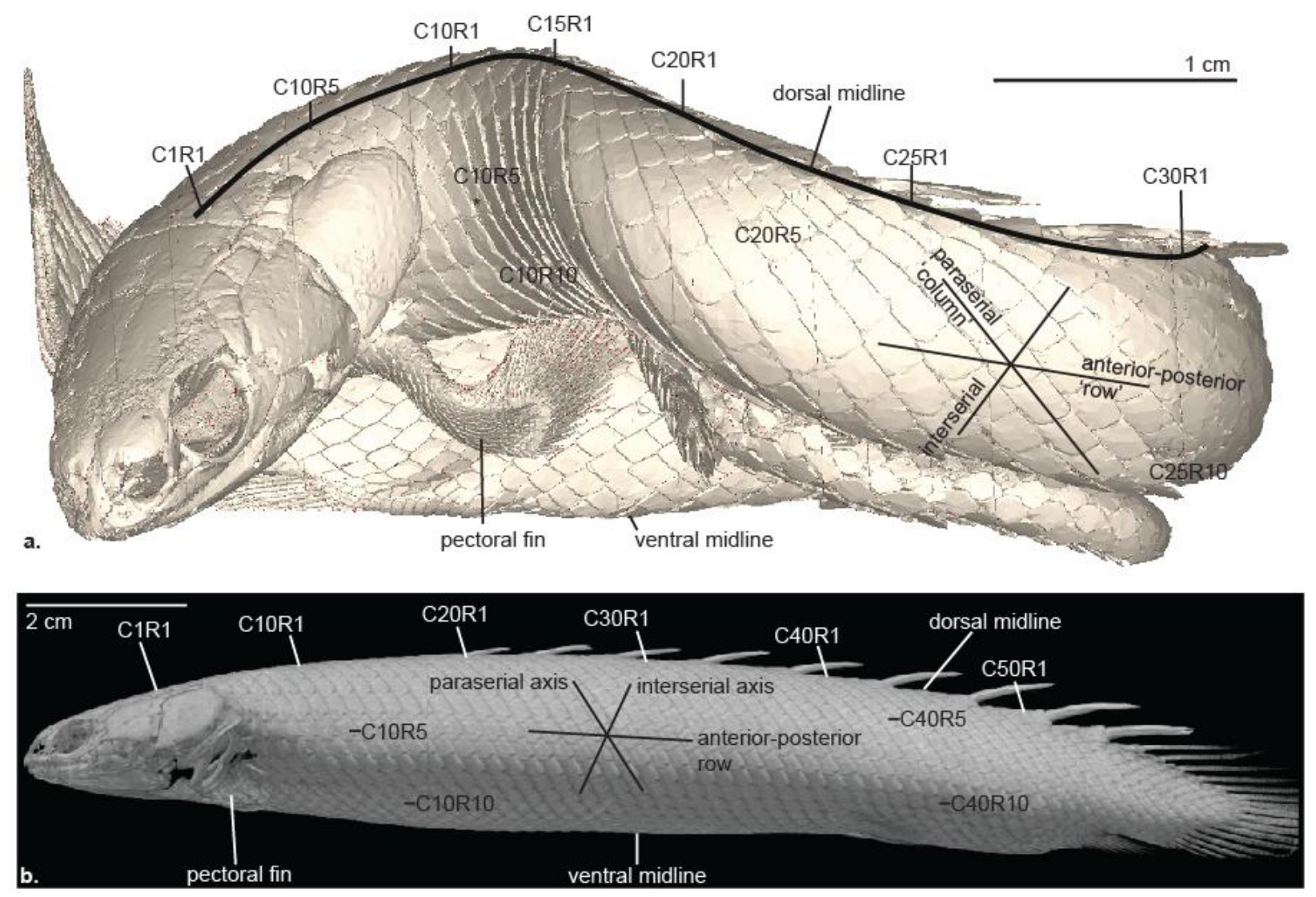

Figure 1. The P. senegalus exoskeleton. (a) $\mu \mathrm{CT}$ reconstruction (18 $\mu \mathrm{m}$ scan resolution) of the $P$. senegalus exoskeleton used in the morphometric analysis, and (b) $\mu \mathrm{CT}$ reconstruction of a $P$. senegalus specimen in a fully extended configuration (95 $\mu \mathrm{m}$ scan resolution), image used with permission from (Humphries, 2003). Images show the paraserial axis of scale articulation (column), the interserial axis of scale overlap, the anterior-posterior row, dorsal and ventral midlines of mirror symmetry, and pectoral fin. Select scales are numbered by column and row number (C\#R\#). The asterisked scale (C9R5) in (a) is used in Fig. 2.

\subsection{Geometric Morphometric Analysis}

A custom-written visual basic script extracted the 3D spatial coordinates of mouse-clicked landmarks (LM) on the STL objects in CAD software (RHINOCEROUS, Robert McNeel and Associates, USA). 

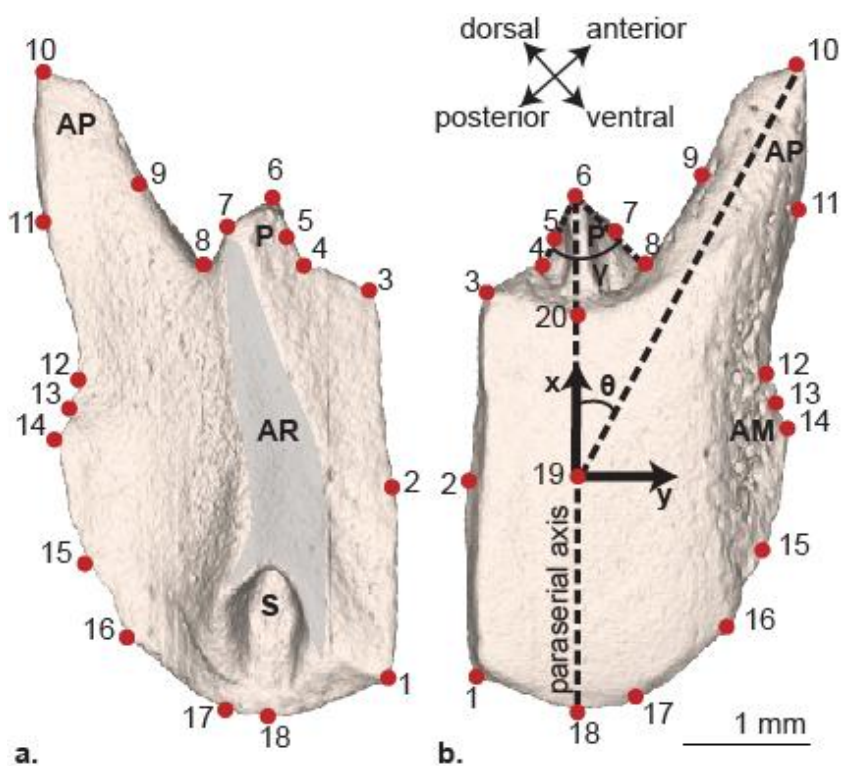

Figure 2. $\mu \mathrm{CT}$ reconstruction (10 $\mu \mathrm{m}$ scan resolution) of a single P. senegalus scale (C9R5), asterisked in Fig. 1a, (a) interior and (b) exterior views. Geometrical features are labeled: peg (P), socket (S), anterior process (AP), axial ridge (AR), and anterior margin (AM). The $20 \mathrm{LMs}$ used for morphometric analysis are shown (red dots) and described in Table 1. The x-y coordinate axis is defined with the $x$-axis aligned with the scale's paraserial axis, and $y$-axis defined $90^{\circ}$ perpendicular to the $\mathrm{x}$-axis in the plane of the scale. Two morphometric parameters are shown (described in Table 2): peg tip angle $(\gamma)$ and anterior process angle $(\theta)$. Dashed lines define edges of angles $\gamma$ and $\theta$. 
Table 1. Landmark typology and descriptions for the 20 LMs defining the 2D scale geometry, illustrated in Fig. 2.

\begin{tabular}{ccl}
\hline Landmark & Type & Description \\
\hline 1 & I & Posterior corner \\
2 & III & Midpoint between dorsal and posterior corners \\
3 & I & Dorsal corner \\
4 & II & Dorsal base of peg \\
5 & III & Midpoint between dorsal base and tip of peg \\
6 & I & Tip of peg \\
7 & III & Midpoint between anterior base and tip of peg \\
8 & II & Anterior base of peg \\
9 & II & Anterior point of anterior margin transition (interserial overlap) \\
10 & I & Tip of anterior process (most anterior point) \\
11 & II & Anterior point of paraserial overlap transition \\
12 & II & Anterior margin valley \\
13 & III & Midpoint between anterior margin valley and anterior margin peak \\
14 & II & Anterior margin peak \\
15 & II & Ventral point of paraserial overlap transition \\
16 & I & Posterior point of anterior margin transition (interserial overlap) \\
17 & I & Ventral corner \\
18 & I & Midpoint of socket on edge of scale \\
19 & II & Point between tip of peg and socket on the top side of scale \\
20 & II & Central base of peg \\
\hline
\end{tabular}

The 18 LMs defining the 2D outline converged on the 2D polygonal area of the scale. Fig. 3 shows a representative scale (C30R10) with 60 LMs numbered in order of their selection to define the outline of a scale; the first $18 \mathrm{LM}$ used for the morphometric analysis are shown in red. Fig. 3b plots the 2D (x-y) polygonal area defined by a given number of LMs for five different scales. The polygonal area did not change significantly with increasing the density of LMs beyond the 18 used to define the outline of the scale in this morphometric analysis. Two additional LMs were chosen to define a central point on the top of the scale in the $+z$ direction close to the origin (LM19 in Fig. 2) and the length of the peg (LM20 in Fig. 2). LM typology was assigned in accordance with Bookstein's (1991) descriptions, where:

(i) Type I LMs have discrete position that are clearly identifiable with minimal possibility of error and can be defined independently from other LMs. They mark distinct vertexes in the scale geometry at LM 1, 3, 6, 10, 16, 17, and 18 . 
(ii) Type II LMs have a possibility of placement error and may be positioned relative to other LMs, e.g. local minima or maxima in curvature. They describe loci that shift in relation to other geometric features, at LMs 4, 8, 9, 11, 12, 14, 15, 19, and 20.

(iii) Type III LMs describe geometric features that may be difficult to identify despite a morphological importance. They define LMs that capture scale shape between Type I and Type II LMs at LMs 2, 5, 7, and 13.

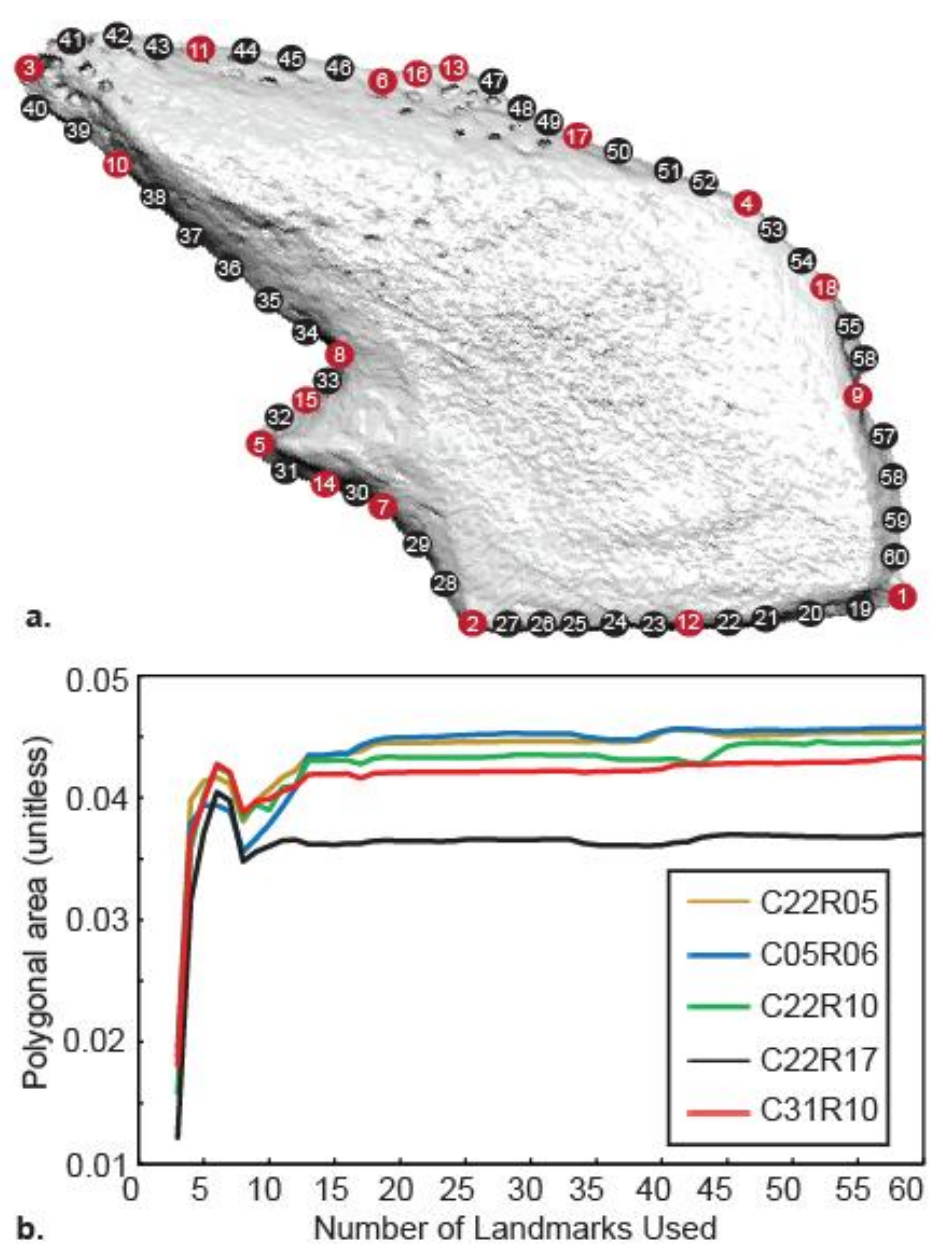

Figure 3. Selection criteria for the number of LM used in the morphometric analysis of P. senegalus scales. (a) Single scale (C31R10) with 60 landmarks placed around outer surface. LMs are numbered in order of their selection for calculating polygonal area. LMs in red represent the 18 LM shown in Fig. 2 and described in Table 1. (b) Plot of polygonal area versus number of landmarks selected to define the outline of the scale, for five different scales in different regions in the $P$. senegalus exoskeleton. 
where $t$ is the grid spacing in both the $\mathrm{x}$ - and y-directions. Axial strains measure length changes along a given direction; for instance, positive and negative values of $\varepsilon_{11}$ correspond to expansion and compression along the $\mathrm{x}$-axis, respectively. Shear strain $\left(\varepsilon_{12}\right)$ measures changes in angles with respect to both $\mathrm{x}$ - and $\mathrm{y}$ axes in plane. The hydrostatic strain for each grid element, representing the strain associated with grid element area change, was computed as: 
$\varepsilon_{\text {hyd }}(m, q)=\frac{1}{2}\left(\varepsilon_{11}(m, q)+\varepsilon_{22}(m, q)\right)$. The deviatoric strain tensor was computed as:

$\varepsilon_{\text {dev }}(m, q)=\left[\begin{array}{cc}\varepsilon_{11}(m, q)-\varepsilon_{\text {hyd }}(m, q) & \varepsilon_{12}(m, q) \\ \varepsilon_{12}(m, q) & \varepsilon_{22}(m, q)-\varepsilon_{h y d}(m, q)\end{array}\right]$, and the Euclidean norm was taken to provide a scalar value representing the shape change of the grid elements. Considering the shape change as plane strain in the $\mathrm{XY}$ plane, the 2D strain energy density for each grid element was calculated as:

$u(m, q)=\frac{1}{2} \sigma^{T} \varepsilon=$ $\frac{1}{2}\left[(2 \mu+\lambda)\left(\varepsilon_{11}(m, q)^{2}+\varepsilon_{22}(m, q)^{2}\right)+2 \lambda\left(\varepsilon_{11}(m, q) * \varepsilon_{22}(m, q)\right)+\mu\left(\gamma_{12}(m, q)^{2}\right)\right]$, where $\mu=$ $\frac{E}{2(1-v)}, \lambda=\frac{E v}{(1+v)(1-2 v)}, \gamma_{12}=2 \varepsilon_{12}$, elastic modulus $E=55 \mathrm{GPa}$ (modulus of ganoine, the outer layer of ganoid scales (Bruet et al., 2008; Song et al., 2011)), and Poisson ratio $v=0.3$. Total strain energy (U) over the 2D transformation grid was calculated as: $U=\sum_{m, q} u(m, q) * \frac{t^{2}}{2}$, a scalar value representing the energy needed to morph the reference geometry into the comparison geometry, essentially describing how different a comparison geometry is from the reference geometry.

To illustrate the meaning of these strain components and their correlation with the deformation of grid elements in the geometric morphometric analysis, we calculated the strain associated with simple affine transformations applied to the reference $P$. senegalus geometry in shape space (compression with equal and opposite extension, simple shear) and form space (scaling) and plotted them as strain contours on their associated transformation grids in Fig. 4. Transformation grids deform uniformly with all grid element edges remaining parallel for affine transformations, and the resulting strain contours are uniform. In compression (Fig. 4b), axial strains are nonzero $\left(\varepsilon_{11}=-\varepsilon_{22}=-0.2\right)$ and shear strain is zero; hydrostatic strain is also zero since there is no grid element area change associated with this deformation, however the deviatoric strain is nonzero $\left(\varepsilon_{d e v}=+0.2\right)$ because the grid elements change shape. In simple shear (Fig. 4c), axial strains are zero while shear strain is nonzero $\left(\varepsilon_{12}=+0.2\right)$; there is no hydrostatic strain as the grid element area does not change, however the grid element shape change causes deviatoric strain to be nonzero $\left(\varepsilon_{d e v}=+0.2\right)$. In uniform scaling (Fig. 4d), axial strains are nonzero $\left(\varepsilon_{11}=\varepsilon_{22}=+0.2\right)$, shear strain 
is zero, hydrostatic strain is nonzero $\left(\varepsilon_{\text {hyd }}=+0.2\right)$ since all grid elements grow in area, and deviatoric strain is zero since no elements deform in shape. Furthermore, we illustrate the gradual morphing of a shape and total strain energy through a series of affine shear operations applied to the reference geometry in Fig. 5. The transformation grids and shear strain plots show that the geometry morphs uniformly with shear operations from $\varepsilon_{12}=-0.5$ to +0.5 . The total strain energy ( $\mathrm{U}$, normalized by maximum value) increases linearly as the grid elements distort with the magnitude of shear. 

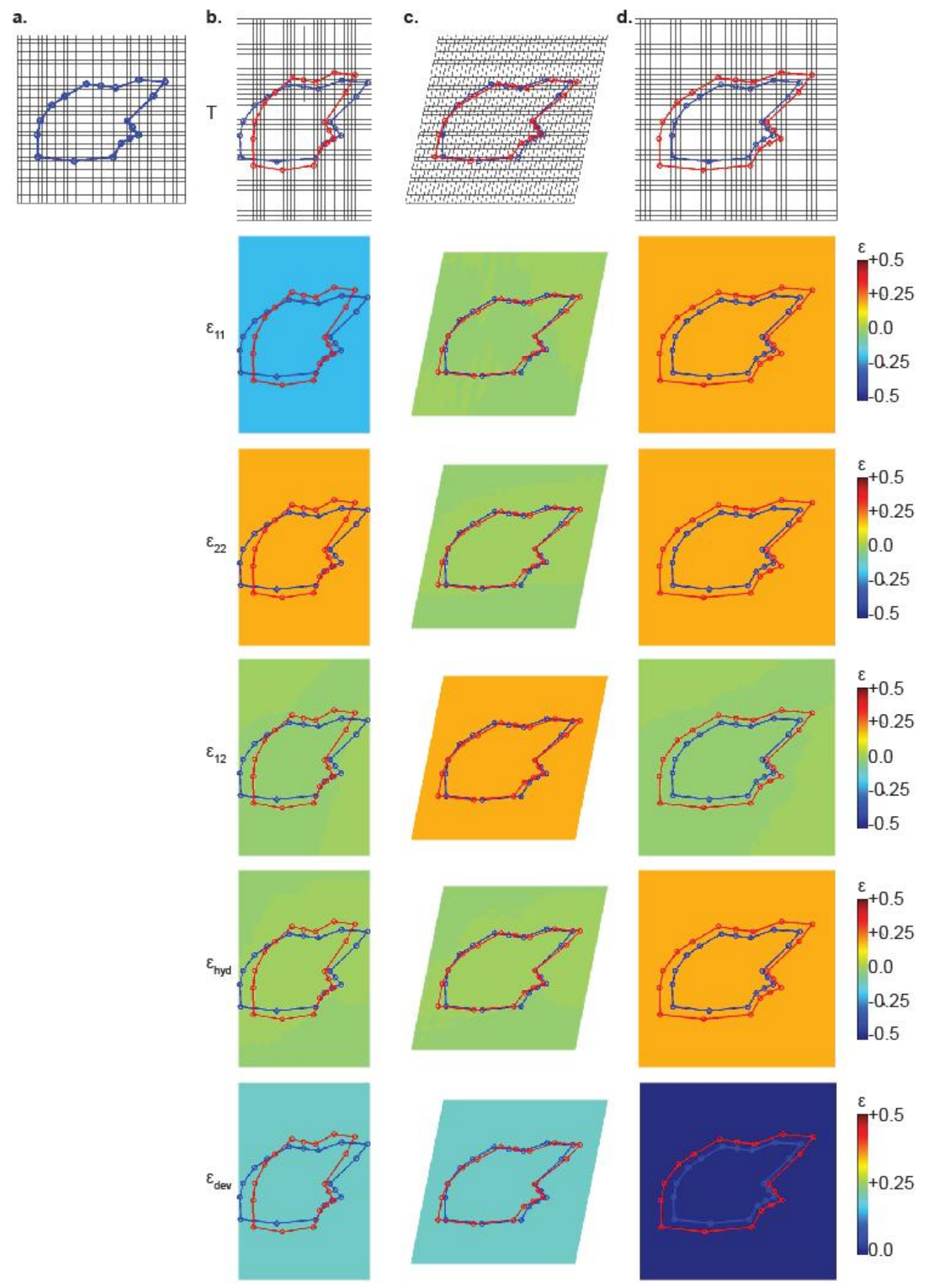
a.

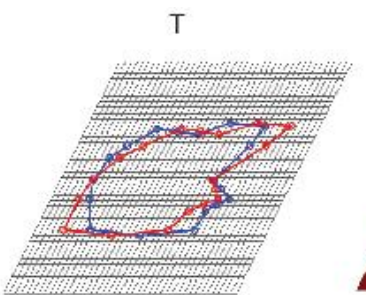

b.

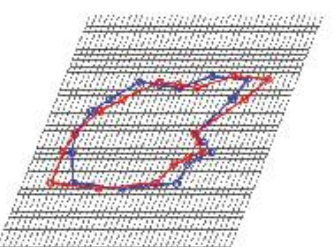

c.

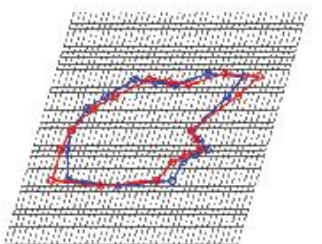

d.

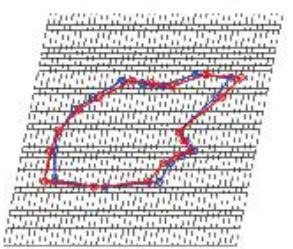

e.

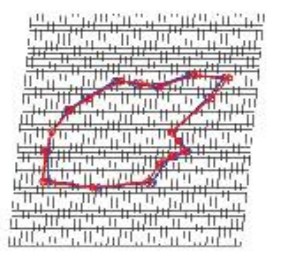

f.

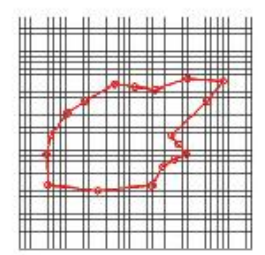

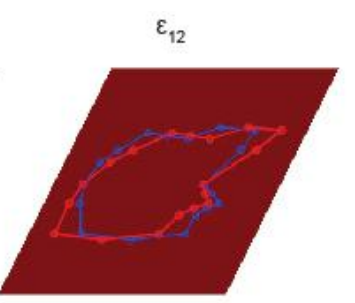
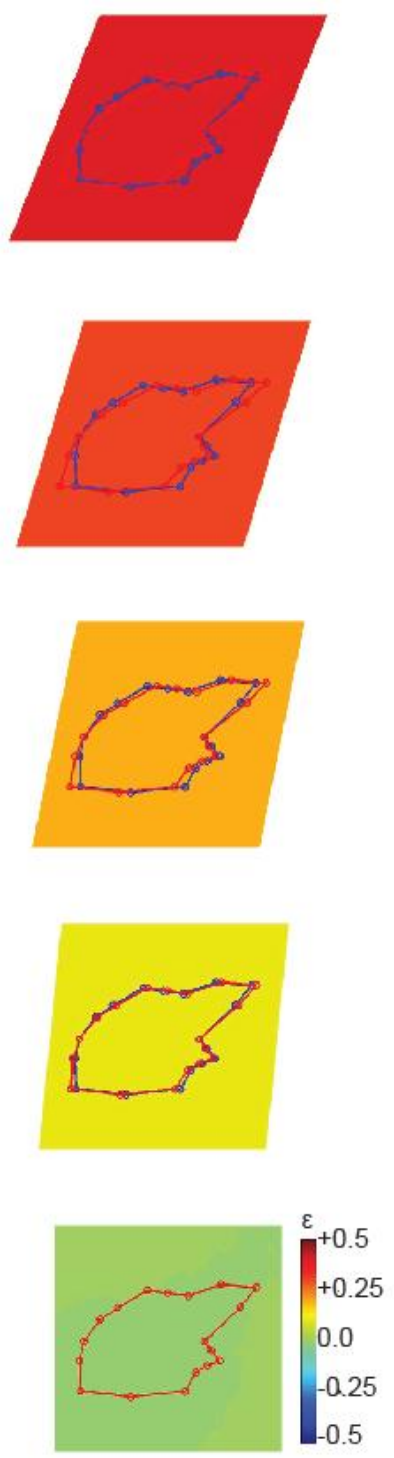

T

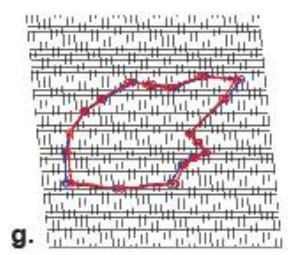

h.
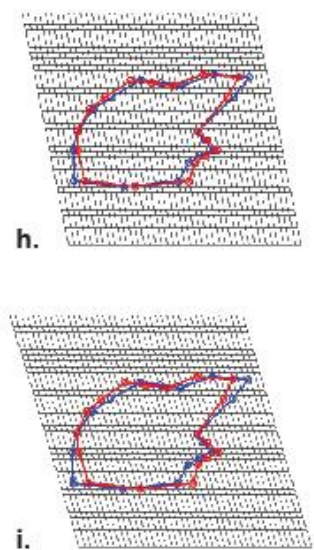

i.
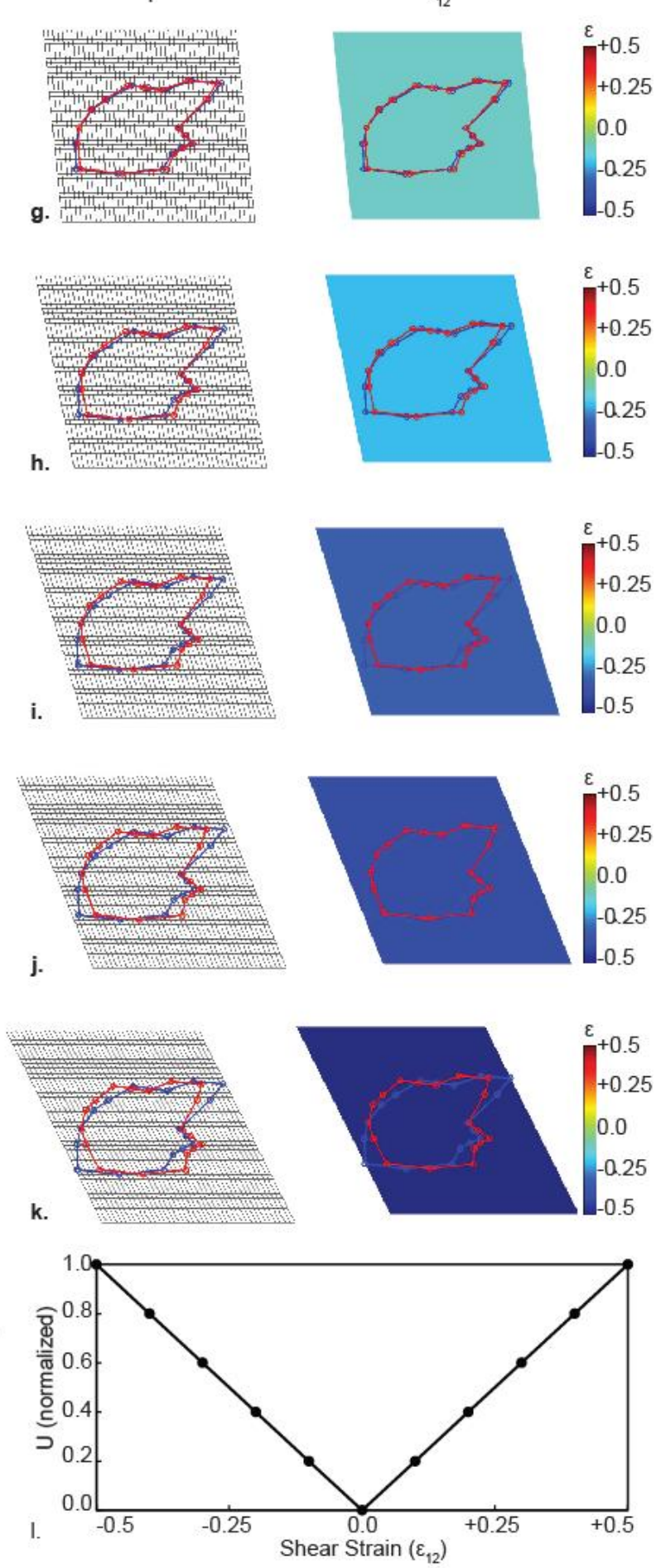

Figure 5. Transformation grids (T) and strain plots mapping the shear component of strain $\left(\varepsilon_{12}\right)$ for a series of affine shear operations (red) applied to the reference geometry (blue): (a) $\varepsilon_{12}=-0.5$, (b) $\varepsilon_{12}=-0.4$, (c) $\varepsilon_{12}=-0.3$, (d) $\varepsilon_{12}=-$ 0.2 , (e) $\varepsilon_{12}=-0.1$, (f) $\varepsilon_{12}=0.0$, (g) $\varepsilon_{12}=+0.1$, (h) $\varepsilon_{12}=+0.2$, (i) $\varepsilon_{12}=+0.3$, (j) $\varepsilon_{12}=+0.4$, (k) $\varepsilon_{12}=+0.5$, and (l) plot of the total strain energy (U, normalized by maximum value) vs. shear strain. 


\subsection{Statistics}

Boxplots of unitless parameter values were generated in MATLAB with the Plotly toolbox. Statistical software (JMP Pro 11, SAS Institute, USA) was used for the student's t-test.

\section{Results}

The mineralized exoskeleton of $P$. senegalus provides full body coverage. The specimen used in this study, shown in the $\mu \mathrm{CT}$ reconstruction in Fig. 1a, has 56 columns and 18 rows of scales identified by column and row number (C\#R\#). Another specimen in an extended configuration (Fig. 1b) illustrates the arrangement of scales in the integument along two interwoven, semi-helical axes: the oblique paraserial axis of scale articulation and the interserial axis of scale overlap (Gemballa \& Bartsch, 2002). The dorsal and ventral midlines, comprised of scales with specialized double-socketed or double-pegged geometries, respectively, represent axes of mirror symmetry in scale geometry and hold the paraserial columns together; these scales are excluded from the subsequent analysis. Armored, limb-like pectoral fins extend from the main body exoskeleton.

The scale geometry is complex. Fig. 2 shows interior and exterior view of a single scale (C9R5) with geometric features highlighted: peg $(\mathrm{P})$ and socket $(\mathrm{S})$ which define the peg-and-socket joint along the paraserial axis, the anterior process (AP) and anterior margin (AM) which form the sliding overlap joint, and the axial ridge (AR) which serves as the site of attachment for the stratum compactum to the dermis. The 2D geometry of the scale is represented by 20 LMs, shown in Fig. 2 and described in Table 1. Five size-independent parameters, listed in Table 2, were calculated from LM coordinates: peg length (PL), AP length (APL), diagonal aspect ratio (DAR), peg tip angle $(\gamma)$, and AP angle $(\theta)$. Scale volume (V) is a size dependent parameter extracted from the $\mu \mathrm{CT}$ reconstruction of the scale. Total strain energy (U) is a size independent parameter calculated from strain formulations. 
Table 2. Morphometric parameters, descriptions, and calculation from landmarks, STL objects, or continuum strain analysis.

\begin{tabular}{lll}
\hline Parameter & Description & Calculation \\
\hline PL & Peg length & Distance between LM6-LM20 \\
APL & Anterior process length & Distance between LM10-LM12 \\
DAR & Diagonal aspect ratio & Distance between LM3-LM16 / Distance between LM1-LM10 \\
$\gamma$ & Peg tip angle & Angle between LM4-LM6-LM8 \\
$\theta$ & Anterior process angle & Angle between LM10-LM19-LM6 \\
V & Volume of scale & Volume of the STL object of the scale \\
$\mathrm{U}$ & Strain energy & $\mathrm{U}=\sum_{m, q} u(m, q) * t^{2}$, where $u(m, q)=\frac{1}{2}\left[(2 \mu+\lambda) *\left(\varepsilon_{11}(\mathrm{~m}, \mathrm{q})^{2}+\right.\right.$ \\
\hline
\end{tabular}

The scale geometry morphs gradually along the anteroposterior axis. Fig. 6a shows $\mu$ CT images of scales across a single row (R6), depicted in the sketch in Fig. 6b. Scale volume (V, normalized by maximum value over all scales) increases from 0.30-1.0 from the anterior region to midsection of the fish (C1R6C24R6) and then decreases to 0.069 in the tail (C25R6-C56R6). The global shape morphs from a complex polygon to a simplified rhombus in the tail. In Fig. 6c, GM transformation grids show the deformation of a square grid fit to the reference geometry (in blue) to generate the biological geometry (in red) of three representative scales from the anterior (C5R6), midsection (C25R6), and tail (C45R6). These plots show that shape change is dominated by shearing of the global geometry as the DAR decreases from 0.69 (C5R6) to 0.59 (C25R6) to 0.54 (C45R6), supplemented by localized compression and expansion around geometric features. 
a.

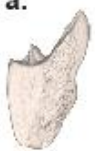

C1R6

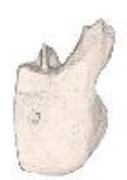

C5R6

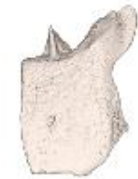

C10R6

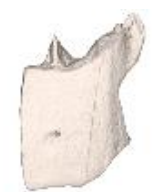

C15R6

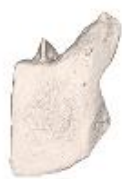

C20R6

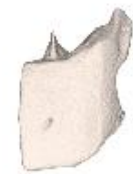

C25R6

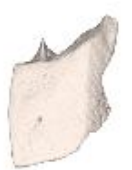

C30R6

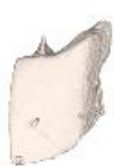

C35R6

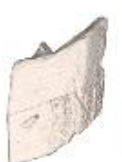

C40R6
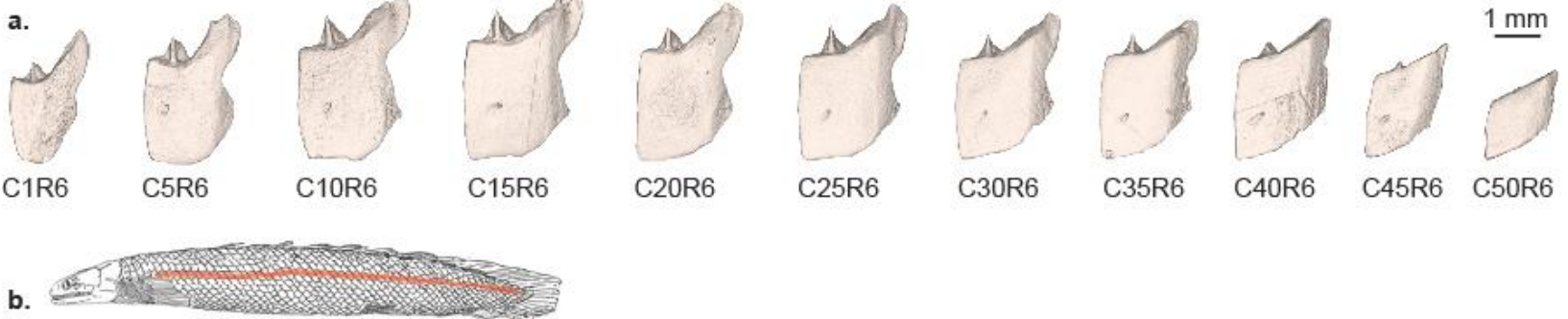

c.

C25R6
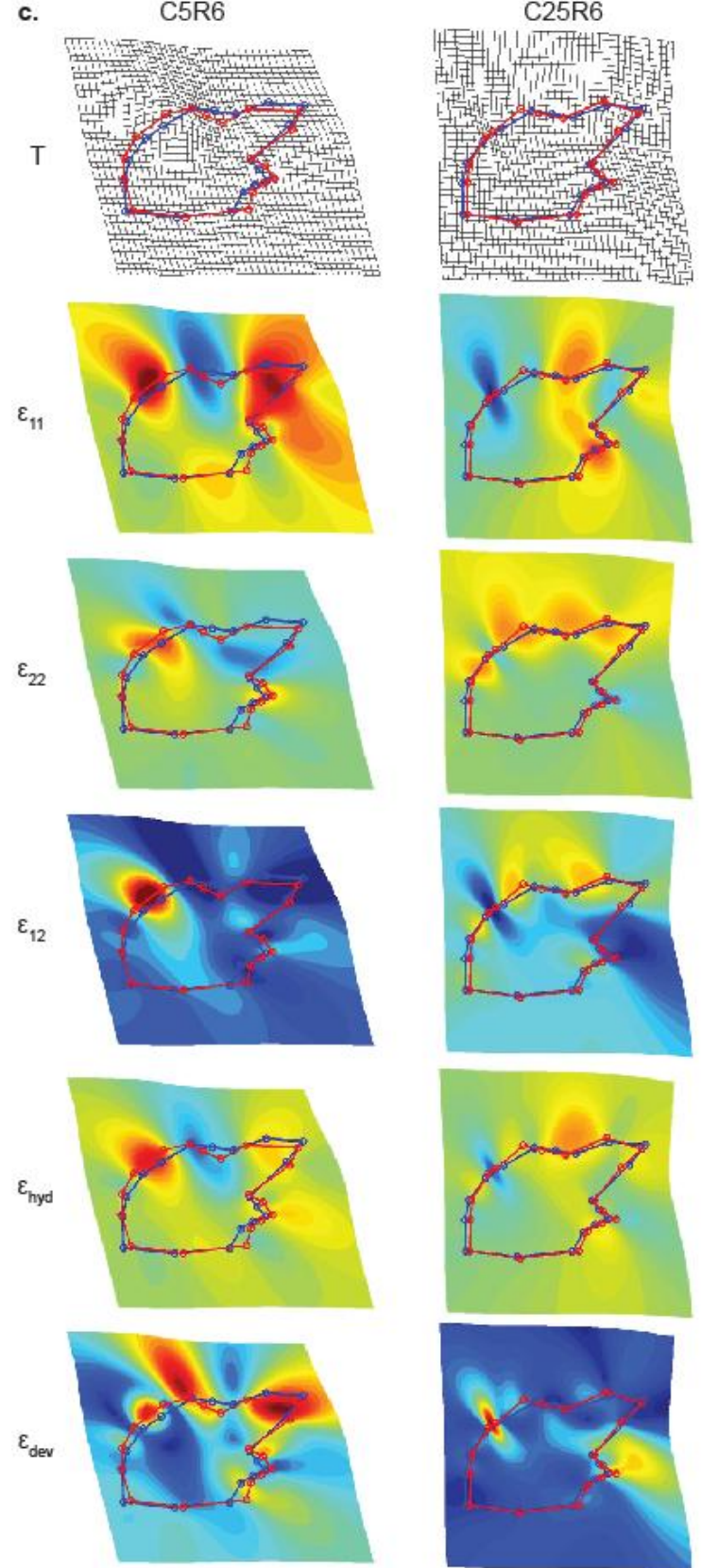

C45R6
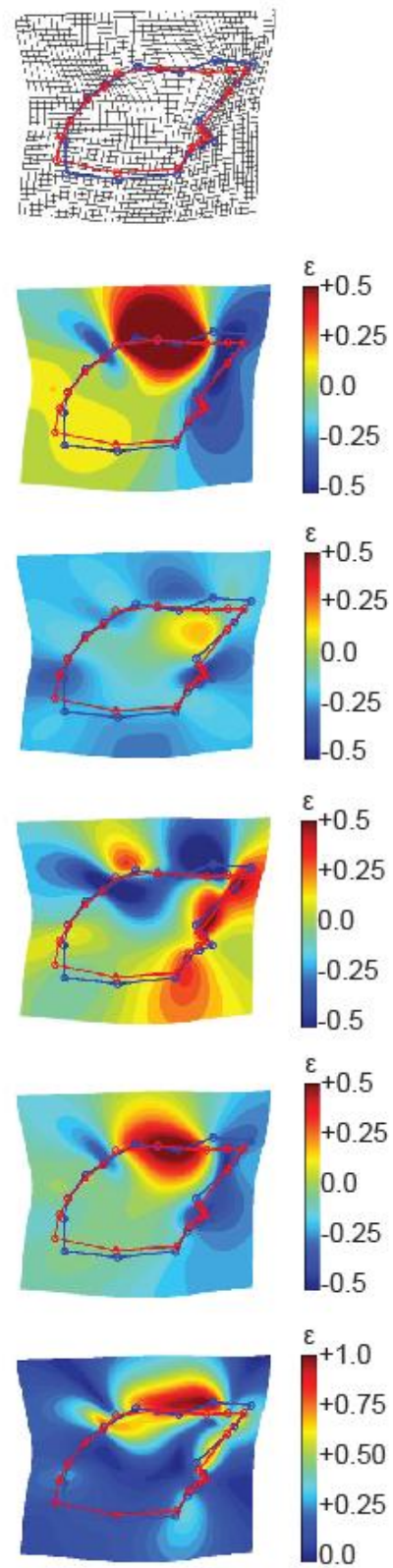

Figure 6. Scale shape variation from anterior to posterior. (a) $\mu \mathrm{CT}$ reconstruction of scales within a single row (R6) of the P. senegalus specimen, every fifth scale shown (15 $\mu \mathrm{m}$ scan resolution). (b) Schematic of the P. senegalus exoskeleton illustrating the row of scales used for the analysis. (c) Transformation grids (T) compare the geometry 
of three scales (C5R6, C25R6, and C45R6; in red) to the reference geometry (blue). Strain plots map the components of the strain tensor $\left(\varepsilon_{11}, \varepsilon_{22}\right.$, and $\left.\varepsilon_{12}\right)$, hydrostatic strain $\left(\varepsilon_{h y d}\right)$ and norm of the deviatoric strain tensor $\left(\varepsilon_{d e v}\right)$ for each grid element.

Components of the $2 \mathrm{D}$ strain tensor $\left(\varepsilon_{11}, \varepsilon_{22}\right.$, and $\left.\varepsilon_{12}\right)$ were calculated for each grid element in the transformation grid and plotted in Fig. 6c to provide enhanced visual contrast of the mechanisms governing shape change. Shear strain $\left(\varepsilon_{12}\right)$ plots show the global shearing phenomenon: for C5R6 the grid shears to the left and $\varepsilon_{12}$ is predominantly negative, C25R6 shows low magnitudes of shear strain, and C45R6 has several regions of high, positive shear strain. Localized regions of axial and shear strain correlate with length scale changes in geometric features of scales. The peg is longer anteriorly with a similar sharpness $\left(\mathrm{PL}=0.12, \gamma=71.7^{\circ}\right.$ for C5R6) compared to the reference geometry, which is closely represented by $\mathrm{C} 25 \mathrm{R} 6\left(\mathrm{PL}=0.11, \gamma=71.4^{\circ}\right)$, while shorter and flatter in the tail $\left(\mathrm{PL}=0.048, \gamma=79.6^{\circ}\right.$ for C45R6), visualized through localized axial and shear strain around the peg. The peg disappears caudally ( $\mathrm{PL}=0.02, \gamma=173^{\circ}$ for C56R6). In anterior scales, the AP has a similar length to the midsection scales $(\mathrm{APL}=0.26$ for $\mathrm{C} 5 \mathrm{R} 6, \mathrm{APL}=0.25$ for $\mathrm{C} 25 \mathrm{R} 6)$, but the concave $\mathrm{AM}$ is more pronounced as visualized by the negative axial strains around LM12 for C5R6. Tail scales have shorter APs and flat AMs (APL $=0.12$ for C45R6), visualized by the negative axial strain around LM10 and positive axial strain around LM12. Orientation of the AP relative to the peg remains constant along the row of scales $(\theta$ $=47.8^{\circ}$ for $\mathrm{C} 5 \mathrm{R} 6, \theta=49.6^{\circ}$ for $\mathrm{C} 25 \mathrm{R} 6, \theta=49.3^{\circ}$ for $\left.\mathrm{C} 45 \mathrm{R} 6\right)$.

Total strain energy (U, normalized by maximum value over all scales) represents the energy needed to morph the reference geometry into the comparison geometry. Lower values of $U$ correspond to scale geometries that are most similar to the reference geometry, e.g. midsection scales (C25R6-C40R6) with U $<0.1$. Anterior scales have $\mathrm{U}$ ranging from $0.1-0.2$, representing geometries that vary moderately from the reference geometry. Caudal scales see U climbing from 0.1-1.0 (C42R6-C56R6) representing geometries with large variance from the reference geometry. Hydrostatic strain $\left(\varepsilon_{\text {hyd }}\right)$ and norm of the deviatoric strain $\left(\varepsilon_{\text {dev }}\right)$ are plotted in Fig. 6c, and show that the anterior scales differ from the average 
scale geometry by AM shape and shearing of the global geometry, while the tail scales differ by the AM and AP shape.

Scale geometry morphs rapidly dorsoventrally through the transformation grids. Fig. 7a shows every other scale down a single, paraserial column of scales (C22) in the specimen's midsection, depicted in the corresponding sketch in Fig. 7b. Scale volume increases from 0.36-0.88 in the dorsal region (C22R2C22R7) and decreases to 0.15 ventrally (C22R8-C22R17). AP length and orientation and AM shape change with scale volume. Global shape change is characterized by a shrinking aspect ratio and the skewing of geometric features relative to each other. Transformation grids for three representative scales (C22R2, C22R8, and C22R14) in Fig. 7c show that scale shape change is dominated by a combination of y-directional compression and shearing of the global geometry as the DAR decreases from 0.60 (C22R2) to 0.58 (C22R8) to 0.36 (C22R14), supplemented by localized compression and expansion. 
a.
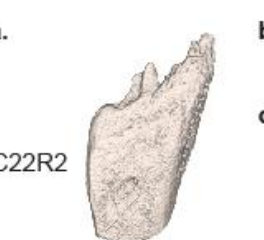

C22R4

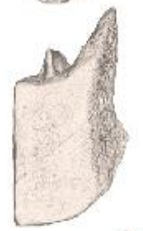

C22R6
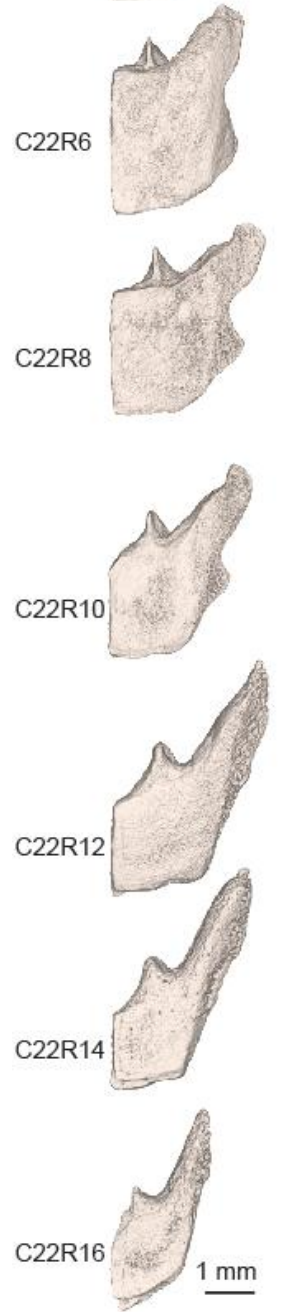

b.

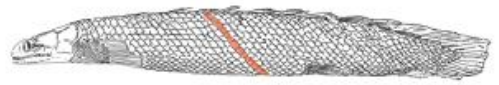

c.
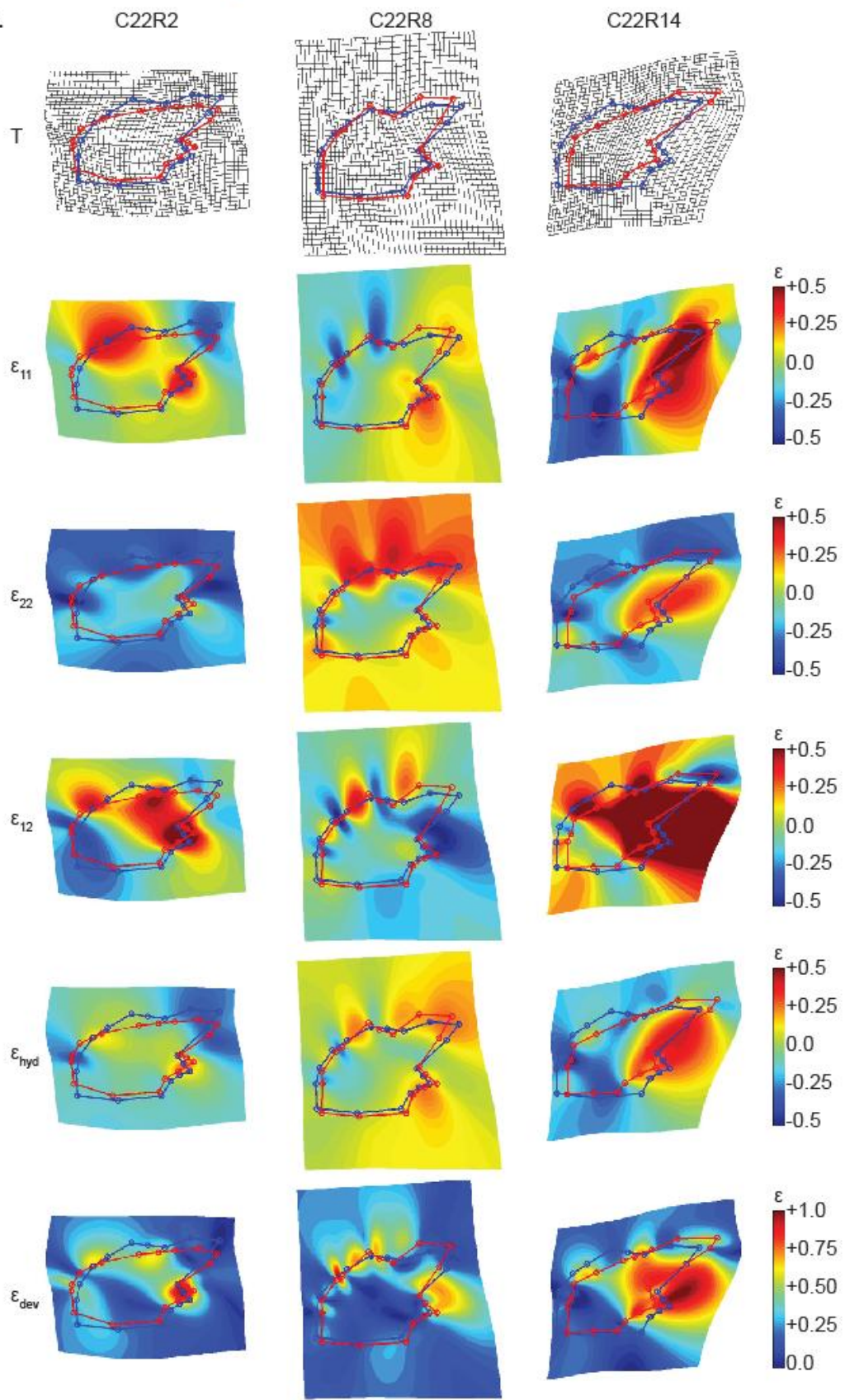

Figure 7. Scale shape variation from dorsal to ventral. (a) $\mu \mathrm{CT}$ reconstruction of scales within a single column (C22) of the $P$. senegalus specimen, every other scale shown (15 $\mu \mathrm{m}$ scan resolution). (b) Schematic of the $P$. senegalus exoskeleton illustrating the column of scales used for the analysis. (c) Transformation grids (T) compare the geometry of three scales (C22R2, C22R8, and C22R14; in red) to the reference geometry (blue). Strain plots map the components of the strain tensor $\left(\varepsilon_{11}, \varepsilon_{22}\right.$, and $\left.\varepsilon_{12}\right)$, hydrostatic strain $\left(\varepsilon_{\text {hyd }}\right)$ and norm of the deviatoric strain tensor $\left(\varepsilon_{d e v}\right)$ at each grid point. 
Strain plots in Fig. 7c show global compression: dorsal (C22R2) and ventral (C22R14) scales are compressed in the $y$-direction compared to the reference geometry, while middle scales (C22R8) are expanded in the y-direction. Localized regions of axial and shear strain correlate with distortion of geometric features. The peg is sharper dorsally $\left(\mathrm{PL}=0.10, \gamma=59.2^{\circ}\right.$ for $\left.\mathrm{C} 22 \mathrm{R} 2\right)$ and flatter ventrally (PL $=0.11, \gamma=69.0^{\circ}$ for $\mathrm{C} 22 \mathrm{R} 8 ; \mathrm{PL}=0.092, \gamma=94.0^{\circ}$ for $\left.\mathrm{C} 22 \mathrm{R} 14\right)$ through localized axial and shear strain about the peg. The socket disappears in ventral scales (interior view of scales not shown). The AP rotates away from the peg as $\theta$ increases from $33.7^{\circ}(\mathrm{C} 22 \mathrm{R} 2)$ to $57.8^{\circ}(\mathrm{C} 22 \mathrm{R} 8)$ to $61.4^{\circ}(\mathrm{C} 22 \mathrm{R} 14)$ while APL increases from 0.18 (C22R2) to 0.26 (C22R8) to 0.28 (C22R14), correlated with positive axial and shear strain in the ventral scale between the peg and AP. The AM shape changes drastically. Middle scales have a more pronounced AM, evidenced by axial and shear strains that alternate between negative and positive values between LM11-LM17. The AM flattens in dorsal and ventral scales. Middle scales (C22R6C22R9) have $\mathrm{U} \leq 0.1$, representing geometries that vary moderately from the reference geometry. Dorsal scales have $\mathrm{U}$ ranging from $0.1-0.2$, while ventral scales have $\mathrm{U}$ ranging from 0.1-0.4. Plots of $\varepsilon_{\mathrm{dev}}$ and $\varepsilon_{\text {hyd }}$ show that C22R2 differs from the average scale geometry by peg and AM shape, C22R8 differs by $\mathrm{AM}$ and AP shape, and C22R14 differs by $\theta$ in addition to peg and AM shape.

The exterior surface of the pectoral fin, shown in Fig. 8a, is covered with mineralized scales. The connection between the main exoskeleton and pectoral fin is fully armored and articulated. A row of seven scales (labeled P1-P7) transitioning from the anterior of the main exoskeleton into the pectoral fin is shown in Fig. 8b. The global shape morphs rapidly over $\sim 7 \mathrm{~mm}$ from a complex geometry to simplified quadrilateral with diminished features. Scale volume decreases from 0.11-0.035 over the seam. The transformation grids for three representative scales (P2, P4, P6) in Fig. 8c show that shape change is dominated by a combination of y-directional expansion and shearing of the global geometry as DAR increases from $0.72(\mathrm{P} 2)$ to $0.75(\mathrm{P} 4)$ to $1.0(\mathrm{P} 6)$, supplemented by localized compression and expansion. 
a.

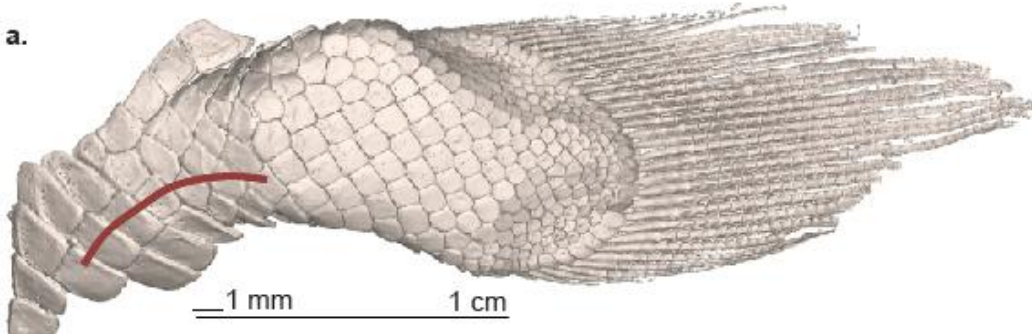

c.

P2
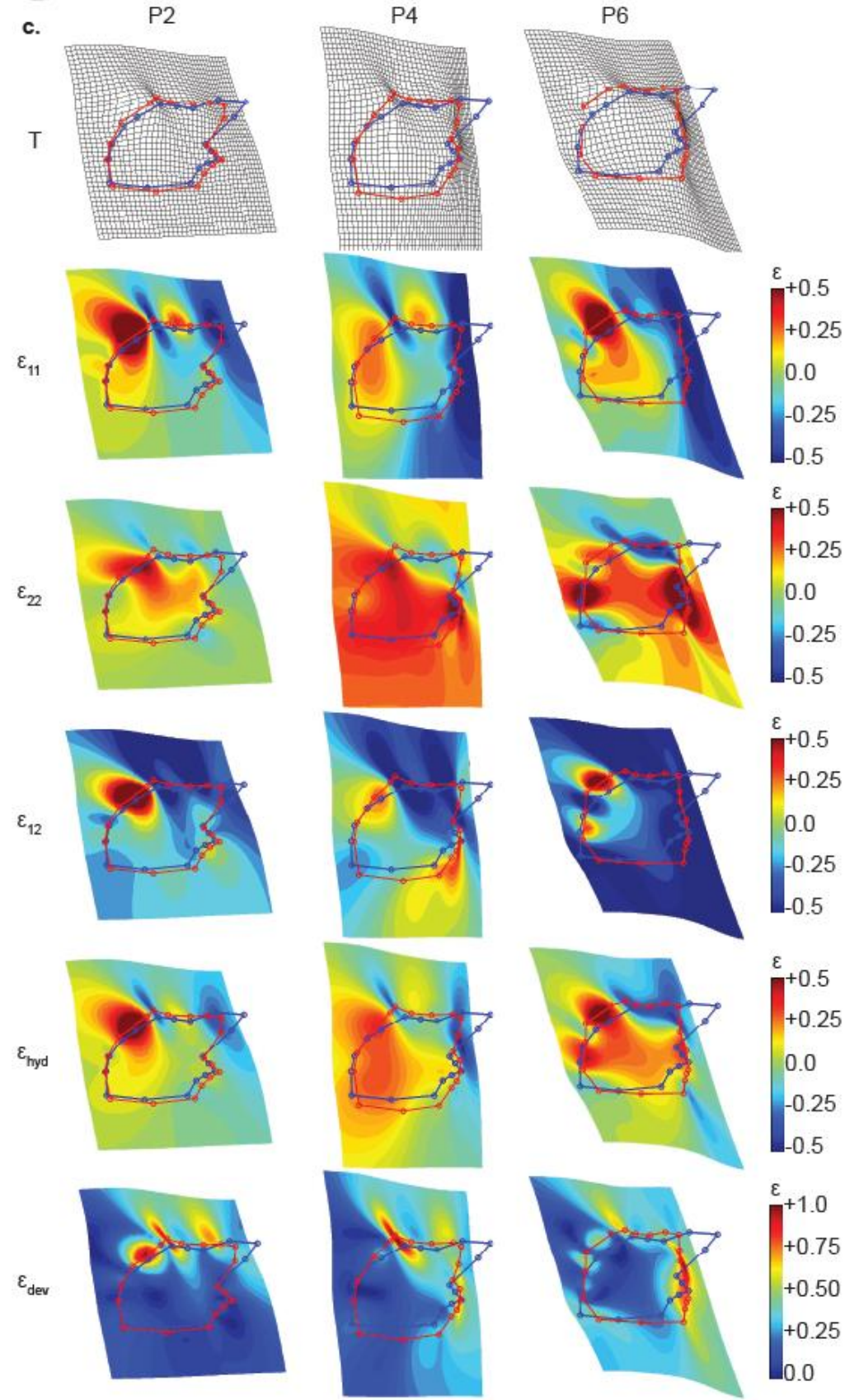

b.

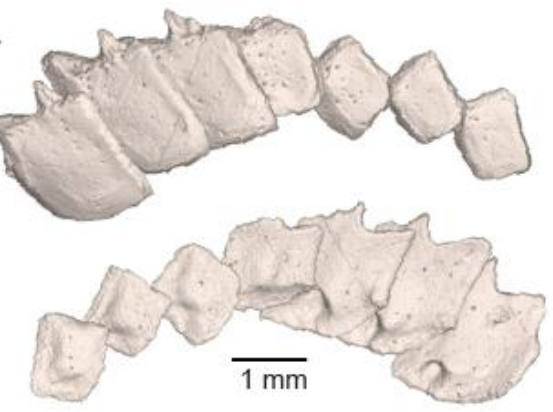


interior (bottom) views of an articulated seam of scales connecting the main exoskeleton to the pectoral fin, highlighted in (a). (c) Transformation grids (T) compare the geometry of three scales (P2, P4, and P6; in red) to the reference geometry (blue). Strain plots map the components of the strain tensor $\left(\varepsilon_{11}, \varepsilon_{22}\right.$, and $\left.\varepsilon_{12}\right)$, hydrostatic strain $\left(\varepsilon_{h y d}\right)$ and norm of the deviatoric strain tensor $\left(\varepsilon_{d e v}\right)$ at each grid point.

Strain plots in Fig. 8c illustrate that the almost-average geometry (P2) first expands in the +y direction (P4), and then shears in the -xy direction (P6). Locally, the peg shortens and flattens over the seam $(\mathrm{PL}=$ 0.098, $\gamma=79.0^{\circ}$ for $\mathrm{P} 2 ; \mathrm{PL}=0.040, \gamma=130^{\circ}$ for $\mathrm{P} 4 ; \mathrm{PL}=0.034, \gamma=148^{\circ}$ for $\mathrm{P} 6$ ) through regional axial and shear strain into an overlap joint and the socket disappears. The AP rotates away from the peg along the seam while the APL remains relatively unchanged $\left(\theta=60.6^{\circ}, \mathrm{APL}=0.14\right.$ for $\mathrm{P} 2 ; \theta=67.1^{\circ}, \mathrm{APL}=$ 0.13 for $\mathrm{P} 4 ; \theta=84.1^{\circ}, \mathrm{APL}=0.12$ for $\left.\mathrm{P} 6\right)$. The AM flattens, shown by alternating negative and positive axial and shear strains between LM11-LM17. Scales in the beginning of the seam (P1-P3) that resemble the average scale geometry have $\mathrm{U}$ ranging from $0.15-0.25$. Remaining scales (P4-P7) have U ranging from 0.3-0.55. Plots of $\varepsilon_{\text {dev }}$ and $\varepsilon_{\text {hyd }}$ show that P2 differs from the average scale geometry by AP and AM shape, P4 differs by AM, AP, and peg shape, and P6 differs by peg and AP shape.

The range of morphometric parameters for the row, column, and pectoral fin seam are plotted as normalized quantities in Fig. 9. The row exhibits the widest spread in all parameter values, perhaps due to containing more scales and spanning a greater distance in the exoskeleton. The column exhibits a wide spread in DAR, V, and U. The seam, despite containing only seven scales with similar volumes, exhibits a wide spread in PL, DAR, $\gamma$, and U. Five zones of scale geometry variants were defined: anterior, average (midsection), tail, ventral, and pectoral fin. Parameter values of scales in these five zones were pooled from the three data sets plus additional scales in the exoskeleton, and the student's t-test was used to determine statistical significance in parameter value differences by zone. P-values from the student's ttest are presented in Table 3. The five scale variants differ from each other through combinations of the morphometric parameters, and we conclude that their morphometries are statistically heterogeneous. 


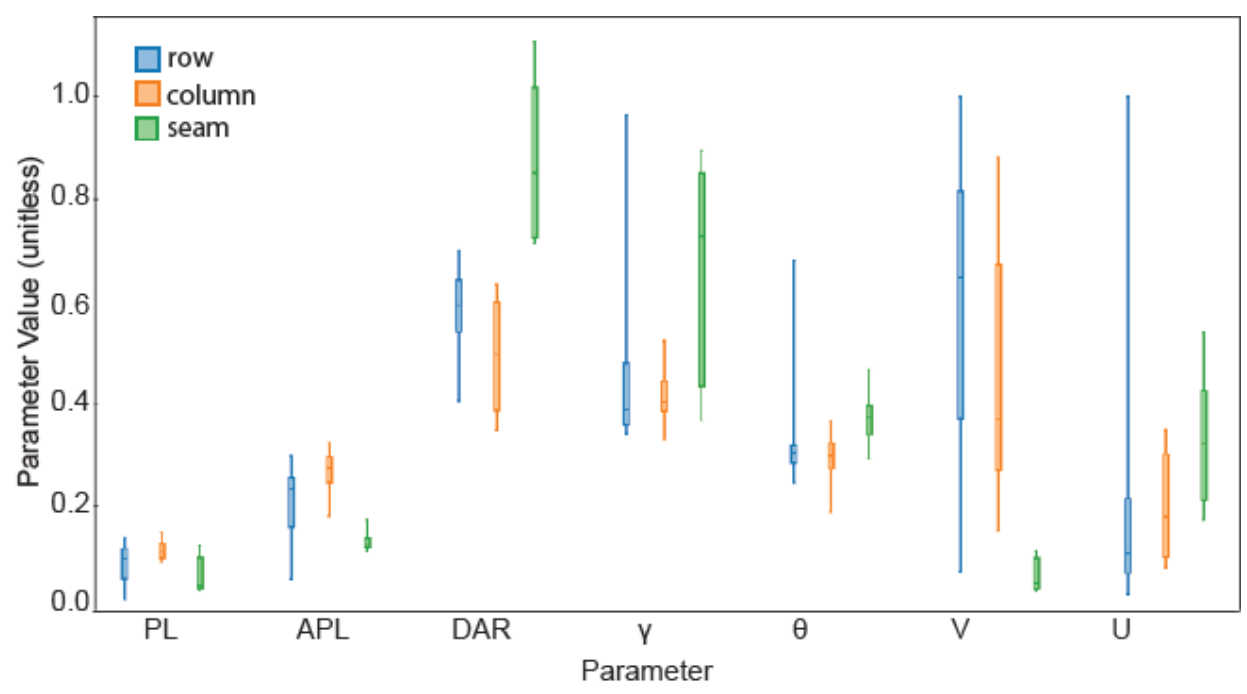

Figure 9. Boxplots for the range of values of morphometric parameters over the row, column, and pectoral fin seam presented in Fig. 6, Fig. 7, and Fig. 8. The calculations for PL, APL, DAR, $\gamma, \theta$, V, and U are described in Table 2. $\gamma$ and $\theta$ are normalized by $180^{\circ}$ to generate unitless parameter values. $\mathrm{V}$ and $\mathrm{U}$ are normalized by their maximum value over all scales $\left(5.44 \mathrm{~mm}^{3}\right.$ and $2.1 \times 10^{10} \mathrm{~J}$, respectively) to generate unitless parameter values. Data are segmented into quartiles with no outliers. 
Table 3. P-values from student's t-test of morphometric parameters of scales grouped by region in the fish exoskeleton: average ( $\mathrm{N}=30$ scales), anterior $(\mathrm{N}=36$ scales $)$, tail $(\mathrm{N}=21$ scales $)$, ventral ( $\mathrm{N}=41$ scales), and pectoral fin $(\mathrm{N}=14$ scales $)$. Asterisks $(*)$ represent $\mathrm{p}$-values $<0.01$.

\begin{tabular}{|c|c|c|c|c|c|}
\hline & & & & \\
\hline & & Anterior & Tail & Ventral & Fin \\
\hline \multirow{7}{*}{$\begin{array}{l}\text { Average } \\
(\mathrm{N}=30)\end{array}$} & $\mathrm{PL}$ & * & * & * & $*$ \\
\hline & APL & 0.90 & * & * & * \\
\hline & DAR & $*$ & $*$ & $*$ & * \\
\hline & $\gamma$ & 0.24 & $*$ & $*$ & $*$ \\
\hline & $\theta$ & 0.87 & $*$ & $*$ & $*$ \\
\hline & V & $*$ & $*$ & $*$ & $*$ \\
\hline & $\mathrm{U}$ & 0.21 & * & * & * \\
\hline \multirow{7}{*}{$\begin{array}{l}\text { Anterior } \\
(\mathrm{N}=36)\end{array}$} & $\mathrm{PL}$ & & $*$ & $*$ & $*$ \\
\hline & APL & & $*$ & * & * \\
\hline & DAR & & $*$ & $*$ & * \\
\hline & $\gamma$ & & * & * & * \\
\hline & $\theta$ & & * & * & * \\
\hline & V & & $*$ & * & * \\
\hline & $\mathrm{U}$ & & * & * & * \\
\hline \multirow{7}{*}{$\begin{array}{c}\text { Tail } \\
(\mathrm{N}=21)\end{array}$} & $\mathrm{PL}$ & & & $*$ & 0.84 \\
\hline & APL & & & $*$ & 0.91 \\
\hline & DAR & & & $*$ & $*$ \\
\hline & $\gamma$ & & & * & $*$ \\
\hline & $\theta$ & & & 0.06 & $*$ \\
\hline & $\mathrm{V}$ & & & 0.80 & $*$ \\
\hline & $\mathrm{U}$ & & & 0.11 & $*$ \\
\hline \multirow{7}{*}{$\begin{array}{l}\text { Ventral } \\
(\mathrm{N}=41)\end{array}$} & $\mathrm{PL}$ & & & & $*$ \\
\hline & APL & & & & $*$ \\
\hline & DAR & & & & $*$ \\
\hline & $\gamma$ & & & & $*$ \\
\hline & & & & & $*$ \\
\hline & $\mathrm{V}$ & & & & $*$ \\
\hline & $\mathrm{U}$ & & & & $*$ \\
\hline
\end{tabular}

\section{Discussion}

The $P$. senegalus integument utilizes heterogeneity in scale morphometry to generate a protective exoskeleton that provides uniform coverage and preserves biomechanical flexibility. The trends in morphometric variation observed in this study are consistent with literature reports along the dorsoventral (Pearson, 1981) and anteroposterior (Gemballa \& Bartsch, 2002) axes. The P. senegalus integument is capable of achieving the same extreme curvature in axial bending in every location along the entire body, however the mechanisms by which these curvatures are achieved vary depending on the morphometry of the scale (Gemballa \& Bartsch, 2002). Constraints on the allowable ranges of motion between $P$. 
senegalus scales are defined by joint type along the paraserial and interserial axes. A schematic of four scales in their native state (straight fish body) are shown in Fig. 10a. The interserial overlap joint allows for sliding (Fig. 10b) as the AM and AP slide under the adjacent scale; sliding is limited by the width of the AR, which serves as the site of connection to the stratum compactum and underlying dermis. The scales can also splay interserially (Fig. 10c) as paraserial columns rotate away from each other. Small paraserial rotations (Fig. 10d) and bending (Fig. 10e) may be allowed within a column of scales, limited by the dorsal (peg and surrounding area) and ventral (socket and surrounding area) surfaces of connection and the collagenous Sharpey's fibers that reinforce, support, and align the joint. We describe the interscale mobility mechanisms that are used by the five scale variants described in the Results:

1. Average scales are large and shaped similar the reference geometry with pronounced features. The peg and socket provide tight articulation for structural rigidity down a column of scales to resist paraserial rotation, paraserial bending, and interserial splay in order to sustain compressive and tensile strains along the paraserial axis (Gemballa \& Bartsch, 2002; Pearson, 1981). The long AP and concave AM allow for interserial sliding in axial bending and torsion. Gemballa and Bartsch (2002) measured the degree of interserial sliding in axial bending on live specimens; they define 'lateral strain and 'lateral stress' as percent change in scale overlap between adjacent scales on concave and convex sides of the body, respectively, normalized to 1.0 representing a straight body (Gemballa \& Bartsch, 2002). When the fish trunk is bent, scales on the concave side of the exoskeleton increase their scale overlap via sliding, while scales on the convex side decrease overlap by sliding apart. Scales in the mid-section of the trunk can achieve lateral strains ranging from 0.61-0.80 and lateral stresses ranging from 1.21-1.24 (Gemballa \& Bartsch, 2002).

2. Anterior scales are smaller than the average scale, and their pure shape is negatively sheared from the average scale. Expansion of the peg reinforces a tight peg-and-socket joint to further restrict paraserial rotation, paraserial bending, and interserial splay enabling the scales to sustain the helical component of torsional strain in the integument (Gemballa \& Bartsch, 2002; Pearson, 1981). The AR is narrower and negative axial and shear strain contours around LM12 yield a 
more pronounced AM alongside a large AP to achieve greater degrees of interserial sliding (lateral strains of 0.42-0.46 and lateral stresses of 1.34-1.40; Gemballa \& Bartsch, 2002), although the fish rarely flexes the anterior region of its trunk during steady state swimming.

3. Tail scales are smaller with a less complex geometry that is positively sheared from the average scale geometry. Negative axial strain contours around LM10 in conjunction with positive axial strain around LM12 yield a short AP, flat AM, and wide AR so that the tail scales achieve small degrees of interserial sliding (lateral strains of 0.92-0.96 and lateral stresses of 1.00-1.01; Gemballa \& Bartsch, 2002). The fish flexes its tail rapid during steady state swimming and achieves high curvatures. Localized axial and shear strain shorten and flatten the peg to relax paraserial rotational and bending constraints, while the smaller subunit size of tail scales allows for smaller radii of curvature before scales touch in the segmented assembly. These geometric modifications allow the tail to accommodate flexure with small changes in scale overlap.

4. Ventral scales are globally compressed in the y-direction and positively sheared from the average geometry. Regional positive axial and shear strains flatten the pronounced pegs without changing their length, so that the peg-and-socket joint continues to restrict paraserial rotation. However, the vanishing socket in ventral scales allows them to paraserially bend and interserially splay within the plane of the integument. Since the plane of the integument in the ventral region of the body is no longer in the same plane as axial bending and torsion, interscale bending and splay are necessary to allow the exoskeleton curve. Alternating negative and positive axial and shear strains between LM10-LM17 lengthen the AP and flatten the AM to assist splay between scales while providing full body coverage during body flexion.

5. Pectoral fin scales are small, quadrilateral, with variable shapes that are globally compressed in the x-direction, expanded in the y-direction, and negatively sheared from the average scale geometry. Complex 3D oscillations of the pectoral fin (Gemballa \& Bartsch, 2002) are enabled by the scale morphometry. Axial expansion and compression of the peg in opposite directions flatten the peg-and-socket joint into a second overlap joint, thereby allowing for sliding in both 
the interserial and paraserial axes while removing restrictions on paraserial rotation and bending. Alternating negative and positive axial and shear strains between LM10-LM17 shorten the AP and flatten the AM to promote small radii of curvature during pectoral fin flexure. The transition between the anterior region of the exoskeleton and the pectoral fin is a materially homogeneous seam that utilizes a rapid morphometric gradient, which allows for uniform coverage and eliminates weak spots that often occur in materially heterogeneous seams.

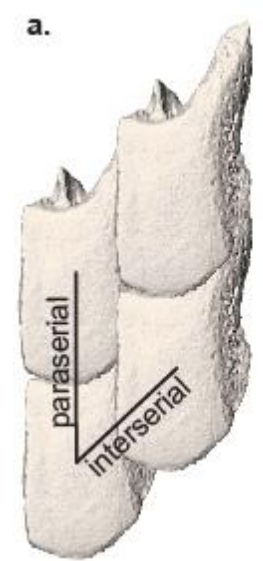

b.

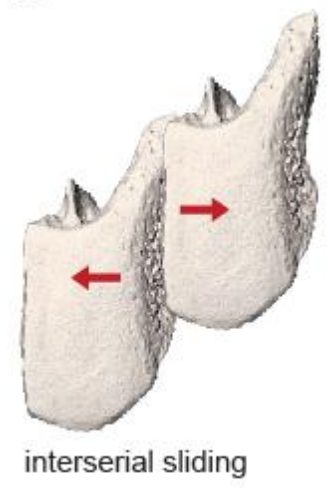

c.

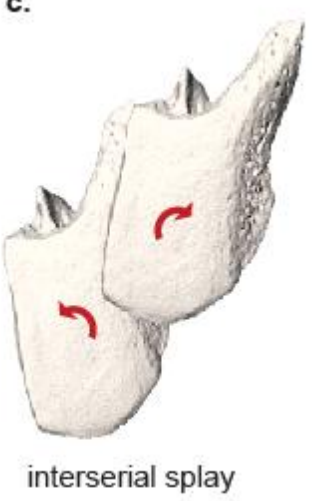

d.

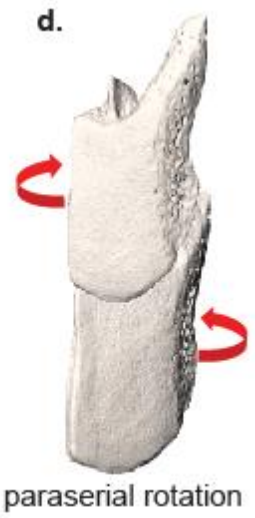

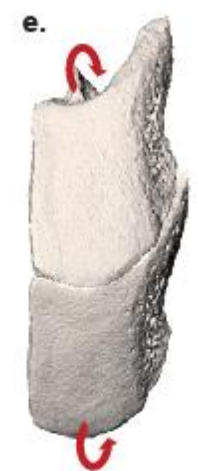

paraserial bending

Figure 10. Schematic of relevant interscale mobility mechanisms. (a) Arrangement of a quad of scales showing interserial and paraserial axis in native (resting) state. Motion of scales can be broken into four components: (b) interserial sliding, (c) interserial splay, (d) paraserial rotation, and (e) paraserial bending.

Our analysis of pure shape change shows how a starting geometry can be stretched or compressed to achieve a new geometry through the application of localized strain fields. This analysis ignores the implications of scale size and orientation discussed by Gemballa \& Bartsch (2002). Landmark-based GM assesses shape variation by comparing landmarks placed on distinct and consistently identifiable anatomical loci on all specimens after removing rigid body transformations (Zelditch et al., 2004; Dryden \& Mardia, 1998; Kendall, 1977; Bookstein, 1991) and then visualizing statistical patterns in the deformation of these loci by fitting thin-plate splines (Bookstein, 1989). Splines are a computationally simple method for curve and surface interpolations (Gueziec, 1996). Unlike smoothing algorithms (e.g. Gaussian) which output a weighted average of pixel distortion around each pixel, splines construct a curve of minimum curvature that passes through a set of reference points and then bend that curve to fit 
the landmarks of a comparison geometry. The warped image of the transformation grids is constructed by interpolating the curves throughout the plane (Little \& Mardia, 1996). The thin-plate spline method provides flexibility to adapt to any suitable subset of landmarks defining a shape, and it is easily extendable from 2D to 3D (Weber, 2011), for instance to study parameters such as thickness, curvature, and angle of overlap in the $P$. senegalus scales.

We assess morphometric heterogeneity using strain plots calculated using continuum element formulations. Recent literature postulated that computing strain may serve as a way to show how and in which ways geometries differ from each other (Weber et al., 2011; Weber, 2011; Bookstein, 2012; Bookstein, 2013). GM deformations are solutions of the biharmonic equation to minimize bending energy, similar to finite element analysis with explicit bending or integrals of the deformation (Bookstein, 2012). By using continuum element formulations to compute 2D strain fields from the thin-plate spline transformations, we use GM to turn observed deformations into computed fictitious forces to better assess how a shape can morph through distinct geometries along a sequence.

To validate our finite element computation of strain, we show that the computed strain fields comparing a biological scale to the reference geometry can be applied as a heterogeneous strain field to the reference geometry to recompose the biological scale geometry. Essentially, a heterogeneous strain field can be applied to a shape in order to morph it into a target geometry. First, we compute the transformation grid and associated strain fields comparing a biological scale (C10R6) to the reference geometry, shown in Fig. 11a. The values for each component of the strain tensor $\left(\varepsilon_{11}, \varepsilon_{22}\right.$, and $\left.\varepsilon_{12}\right)$ at each landmark are extracted. Then, we return to the reference geometry mapped onto an undeformed grid in Fig. 11b and apply the components of the heterogeneous strain field to the landmarks of the reference geometry. After applying all three components, the strained reference geometry is equivalent to the target biological geometry. We conclude that fitting thin-plate splines to deform a reference geometry to a comparison geometry is mathematically equivalent to applying the heterogeneous strain field. 
a.
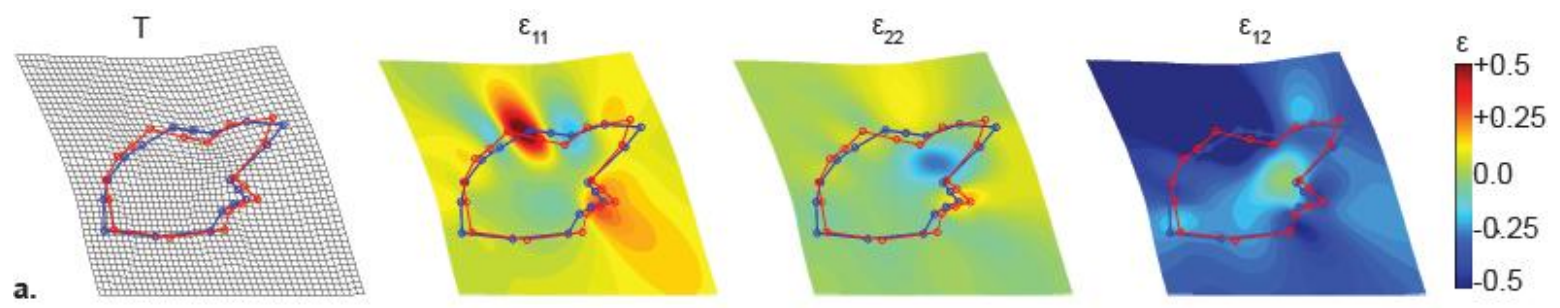

b.
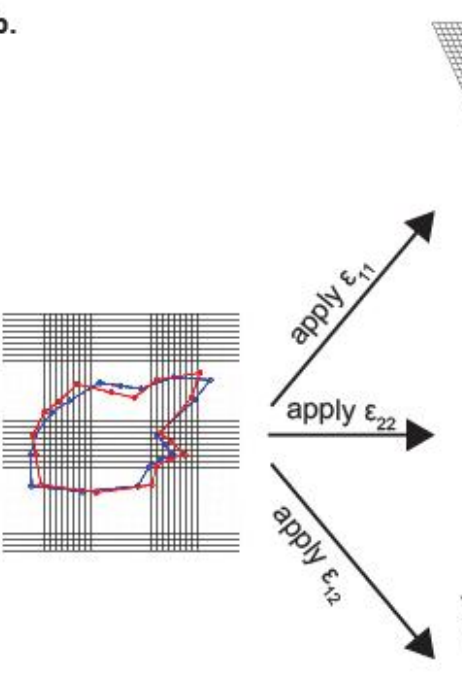

$$
\begin{aligned}
& \text { - Reference geometry } \\
& \text { - Biological geometry } \\
& \text { - Strained geometry }
\end{aligned}
$$
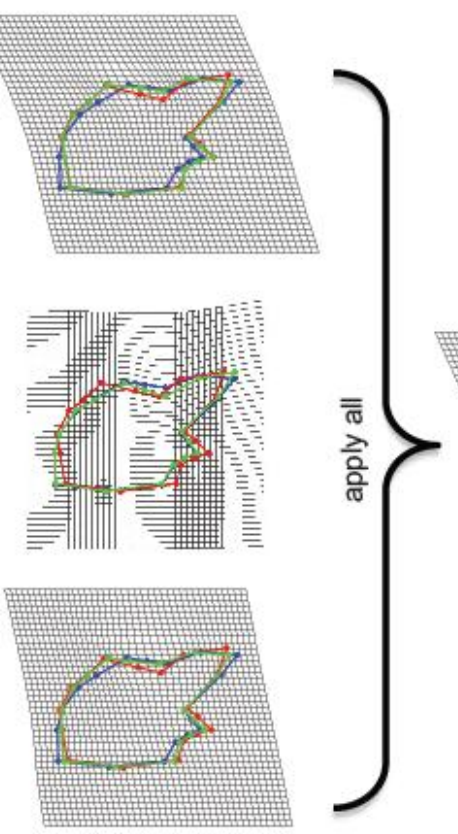

Figure 11. Recomposing morphometric variation by applying strain to a geometry. (a) Transformation grid (T) and strain tensor components $\left(\varepsilon_{11}, \varepsilon_{22}\right.$, and $\left.\varepsilon_{12}\right)$ comparing a biological scale geometry (C10R6, in red) to the reference geometry (blue). (b) Transformation grids resulting from the application of each component of the strain tensor $\left(\varepsilon_{11}\right.$, $\varepsilon_{22}$, and $\varepsilon_{12}$ ) found in $(a)$ to the reference geometry, and transformation grid resulting from the application of all three components of the strain tensor to the reference geometry to recover the geometry of the biological scale.

\section{Conclusion}

The $P$. senegalus exoskeleton combines geometry-based and materials-based structural design principles to generate a protective structure that provides uniform coverage and preserves biomechanical flexibility. In this study, landmark-based GM was used to analyze the spatial morphometric variation of $P$. senegalus scales. Transformation grids based on thin-plate splines visualize the difference in scale geometries. 2D strain plots based on continuum mechanics formulations further illustrate the mechanisms by which geometries morph from each other. Total strain energy provides a quantitative basis to determine how 
different given geometries are from each other. Geometric heterogeneity in scale shape divides the $P$. senegalus exoskeleton into five variants (average, anterior, tail, ventral, and pectoral fin) which utilize different shape-based joint articulation mechanism to move relative to each other within the integument. The technique can also be used to morph a shape into a target geometry with the application of a heterogeneous strain field. In the future, this technique may be used to analyze the geometry change and concomitant changes in interscale mobility mechanisms during the fish's growth and development, for instance comparing the juvenile elasmoid scales of P. senegalus to the adult ganoid scales (Sire, 1989).

The results can be used to develop computational shape morphing algorithms such as our MetaMesh model (Duro-Royo et al., 2014), which defines local, regional, and global hierarchies of design rules of an articulated assembly of $P$. senegalus-inspired sub-units that adapts to complex hosting surfaces. We postulate that shape and shape variation can be used as materials design parameters to tailor the mechanical behavior of materials. Our concurrent work fabricates flexible composite prototypes based on the $P$. senegalus exoskeleton and experimentally characterizes their mechanical behavior. Our future work also seeks to integrate both material heterogeneity, as explored by Araya et al. (2013), and geometric heterogeneity into armor materials that are tailorable to the needs of a wide variety of protective applications, such as custom fit, user mobility, and flexibility around joints.

\section{Conflict of interest statement}

The authors have no financial and personal relationships with other people or organizations that could influence their work.

\section{Acknowledgements}

The authors thank the Orthopedic Biomechanics group at the Beth Israel Deaconess Medical Center for the use of their $\mu \mathrm{CT}$ instrumentation. The authors gratefully acknowledge Steffen Reichert for developing the first iteration of the landmark placement script. This research was funded in part by the National 
Science Foundation Graduate Research Fellowship under grant 1122374, the Institute for Collaborative Biotechnologies through grant W911NF-09-0001 from the U.S. Army Research Office, the Institute for Soldier Nanotechnologies through grant W911NF-13-D-0001 from the U.S. Army Research Office, and the National Security Science and Engineering Faculty Fellowship Program through grant N00244-09-10064. The content of the information does not necessarily reflect the position or the policy of the Government, and no official endorsement should be inferred. 


\section{Table and Figure Captions}

Figure 1. The $P$. senegalus exoskeleton. (a) $\mu \mathrm{CT}$ reconstruction (18 $\mu \mathrm{m}$ scan resolution) of the $P$. senegalus exoskeleton used in the morphometric analysis, and (b) $\mu \mathrm{CT}$ reconstruction of a $P$. senegalus specimen in a fully extended configuration ( $95 \mu \mathrm{m}$ scan resolution), image used with permission from (Humphries, 2003). Images show the paraserial axis of scale articulation (column), the interserial axis of scale overlap, the anterior-posterior row, dorsal and ventral midlines of mirror symmetry, and pectoral fin. Select scales are numbered by column and row number (C\#R\#). The asterisked scale (C9R5) in (a) is used in Fig. 2.

Figure 2. $\mu \mathrm{CT}$ reconstruction (10 $\mu \mathrm{m}$ scan resolution) of a single $P$. senegalus scale (C9R5), asterisked in Fig. 1a, (a) interior and (b) exterior views. Geometrical features are labeled: peg (P), socket (S), anterior process (AP), axial ridge (AR), and anterior margin (AM). The $20 \mathrm{LMs}$ used for morphometric analysis are shown (red dots) and described in Table 1. The $x-y$ coordinate axis is defined with the $x$-axis aligned with the scale's paraserial axis, and y-axis defined $90^{\circ}$ perpendicular to the $\mathrm{x}$-axis in the plane of the scale. Two morphometric parameters are shown (described in Table 2): peg tip angle $(\gamma)$ and anterior process angle $(\theta)$. Dashed lines define edges of angles $\gamma$ and $\theta$.

Figure 3. Selection criteria for the number of LM used in the morphometric analysis of $P$. senegalus scales. (a) Single scale (C31R10) with 60 landmarks placed around outer surface. LMs are numbered in order of their selection for calculating polygonal area. LMs in red represent the 18 LM shown in Fig. 2 and described in Table 1. (b) Plot of polygonal area versus number of landmarks selected to define the outline of the scale, for five different scales in different regions in the P. senegalus exoskeleton.

Figure 4. Landmark-based GM and continuum strain analysis of affine transformations. (a) Reference scale geometry (blue) mapped to an undeformed grid. (b-d) Transformation grids (T) and strain plots mapping the components of strain tensor $\left(\varepsilon_{11}, \varepsilon_{22}\right.$, and $\left.\varepsilon_{12}\right)$, hydrostatic strain $\left(\varepsilon_{\text {hyd }}\right)$ and the norm of the deviatoric strain tensor $\left(\varepsilon_{\text {dev }}\right)$ for affine transformations (red) applied to the reference geometry (blue): (b) compression $\left(\varepsilon_{11}=-\varepsilon_{22}=-0.2\right)$, (c) simple shear $\left(\varepsilon_{12}=+0.2\right)$ and (d) uniform scaling $\left(\varepsilon_{11}=\varepsilon_{22}=+0.2\right)$.

Figure 5. Transformation grids $(\mathrm{T})$ and strain plots mapping the shear component of strain $\left(\varepsilon_{12}\right)$ for a series of affine shear operations (red) applied to the reference geometry (blue): (a) $\varepsilon_{12}=-0.5$, (b) $\varepsilon_{12}=$ 0.4 , (c) $\varepsilon_{12}=-0.3$, (d) $\varepsilon_{12}=-0.2$, (e) $\varepsilon_{12}=-0.1$, (f) $\varepsilon_{12}=0.0$, (g) $\varepsilon_{12}=+0.1$, (h) $\varepsilon_{12}=+0.2$, (i) $\varepsilon_{12}=+0.3$, (j) $\varepsilon_{12}$ 
$=+0.4$, (k) $\varepsilon_{12}=+0.5$, and (l) plot of the total strain energy (U, normalized by maximum value) vs. shear strain.

Figure 6. Scale shape variation from anterior to posterior. (a) $\mu \mathrm{CT}$ reconstruction of scales within a single row (R6) of the P. senegalus specimen, every fifth scale shown (15 $\mu \mathrm{m}$ scan resolution). (b) Schematic of the $P$. senegalus exoskeleton illustrating the row of scales used for the analysis. (c) Transformation grids (T) compare the geometry of three scales (C5R6, C25R6, and C45R6; in red) to the reference geometry (blue). Strain plots map the components of the strain tensor $\left(\varepsilon_{11}, \varepsilon_{22}\right.$, and $\left.\varepsilon_{12}\right)$, hydrostatic strain $\left(\varepsilon_{h y d}\right)$ and norm of the deviatoric strain tensor $\left(\varepsilon_{d e v}\right)$ for each grid element.

Figure 7. Scale shape variation from dorsal to ventral. (a) $\mu \mathrm{CT}$ reconstruction of scales within a single column (C22) of the P. senegalus specimen, every other scale shown (15 $\mu \mathrm{m}$ scan resolution). (b) Schematic of the P. senegalus exoskeleton illustrating the column of scales used for the analysis. (c) Transformation grids (T) compare the geometry of three scales (C22R2, C22R8, and C22R14; in red) to the reference geometry (blue). Strain plots map the components of the strain tensor $\left(\varepsilon_{11}, \varepsilon_{22}\right.$, and $\left.\varepsilon_{12}\right)$, hydrostatic strain $\left(\varepsilon_{h y d}\right)$ and norm of the deviatoric strain tensor $\left(\varepsilon_{d e v}\right)$ at each grid point.

Figure 8. The P. senegalus pectoral fin and scale shape variation along a transition seam. (a) $\mu \mathrm{CT}$ reconstruction of the specimen's left pectoral fin ( $8 \mu \mathrm{m}$ scan resolution) highlighting a transition row segment. (b) Exterior (top) and interior (bottom) views of an articulated seam of scales connecting the main exoskeleton to the pectoral fin, highlighted in $(a)$. (c) Transformation grids $(\mathrm{T})$ compare the geometry of three scales (P2, P4, and P6; in red) to the reference geometry (blue). Strain plots map the components of the strain tensor $\left(\varepsilon_{11}, \varepsilon_{22}\right.$, and $\left.\varepsilon_{12}\right)$, hydrostatic strain $\left(\varepsilon_{h y d}\right)$ and norm of the deviatoric strain tensor $\left(\varepsilon_{d e v}\right)$ at each grid point.

Figure 9. Boxplots for the range of values of morphometric parameters over the row, column, and pectoral fin seam presented in Fig. 6, Fig. 7, and Fig. 8. The calculations for PL, APL, DAR, $\gamma, \theta, \mathrm{V}$, and $\mathrm{U}$ are described in Table 2. $\gamma$ and $\theta$ are normalized by $180^{\circ}$ to generate unitless parameter values. $\mathrm{V}$ and $\mathrm{U}$ are normalized by their maximum value over all scales $\left(5.44 \mathrm{~mm}^{3}\right.$ and $2.1 \times 10^{10} \mathrm{~J}$, respectively) to generate unitless parameter values. Data are segmented into quartiles with no outliers.

Figure 10. Schematic of relevant interscale mobility mechanisms. (a) Arrangement of a quad of scales showing interserial and paraserial axis in native (resting) state. Motion of scales can be broken into four components: (b) interserial sliding, (c) interserial splay, (d) paraserial rotation, and (e) paraserial bending. 
Figure 11. Recomposing morphometric variation by applying strain to a geometry. (a) Transformation grid $(\mathrm{T})$ and strain tensor components $\left(\varepsilon_{11}, \varepsilon_{22}\right.$, and $\left.\varepsilon_{12}\right)$ comparing a biological scale geometry (C10R6, in red) to the reference geometry (blue). (b) Transformation grids resulting from the application of each component of the strain tensor $\left(\varepsilon_{11}, \varepsilon_{22}\right.$, and $\left.\varepsilon_{12}\right)$ found in $(a)$ to the reference geometry, and transformation grid resulting from the application of all three components of the strain tensor to the reference geometry to recover the geometry of the biological scale.

Table 1. Landmark typology and descriptions for the 20 LMs defining the 2D scale geometry, illustrated in Fig. 2.

Table 2. Morphometric parameters, descriptions, and calculation from landmarks, STL objects, or continuum strain analysis.

Table 3. P-values from student's t-test of morphometric parameters of scales grouped by region in the fish exoskeleton: average $(\mathrm{N}=30$ scales $)$, anterior $(\mathrm{N}=36$ scales $)$, tail $(\mathrm{N}=21$ scales $)$, ventral $(\mathrm{N}=41$ scales $)$, and pectoral fin $(\mathrm{N}=14$ scales $)$. Asterisks $(*)$ represent $\mathrm{p}$-values $<0.01$. 


\section{References}

Araya, S., Zolotovsky, K., Veliz, F., Song, J., Boyce, M.C., Ortiz, C., 2013. Bioinformed performative composite structures. Education and research in Computer Aided Architectural Design in Europe, 31, $575-84$.

Arciszewski, T., Cornell, J., 2006. Bio-Inspiration: Learning Creative Design Principia. In: Smith, I.D.C (Ed.), Intelligent Computing in Engineering and Architecture. Springer, Berlin, pp. 32-53.

Barbakadze, N., Enders, S., Gorb, S., Arzt, E., 2006. Local mechanical properties of the head articulation cuticle in the beetle Pachnoda marginata (Coleoptera, Scarabaeidae). Journal of Experimental Biology, $209,722-730$.

Bookstein, F.L., 1989. Principle warps: Thin-plate splines and the decomposition of deformations. IEEE Transactions on Pattern Analysis and Machine Intelligence, 2, 567-585.

Bookstein, F.L. (ed.), 1991. Morphometric Tools for Landmark Data: Geometry and Biology. Cambridge University Press, Cambridge.

Bookstein, F.L., 2012. Geometric Morphometrics and Finite Element Analysis: First Results from a Joint Formalism for Modeling Strain. Mathematical Modelling, 7, 78-83.

Bookstein F.L., 2013. Allometry for the twenty-first century. Biological Theory, 7, 10-25.

Brainerd, E.L., 1994. Mechanical design of polypterid fish integument for energy storage during recoil aspiration. Journal of Zoology, 232, 7-19.

Browning, A., Ortiz, C., Boyce, M. C., 2013. Mechanics of composite elasmoid fish scale assemblies and their bioinspired analogues, Journal of the Mechanical Behavior of Biomedical Materials, 19, 75-86.

Bruet, B.J.F., Qi, H. J., Boyce, M.C., Panas, R. Tai, K., Frick, L., Ortiz, C., 2005.. Nanoscale morphology and indentation of individual nacre tablets from the gastropod mollusc Trochus niloticus. Journal of Materials Research, 20, 2400-2419. 
Bruet, B. J. F., Song, J. H., Boyce, M. C., Ortiz, C., 2008. Materials design principles of ancient fish armour. Nature Materials, 7, 748-756.

Chen, P. Y., McKittrick, J., Meyers, M. A., 2012. Biological materials: functional adaptations and bioinspired designs. Progress in Materials Science, 57, 1492-1704.

Connors, M., Ehrlich, H., Hog, M., Godeffroy, C., Araya, S., Kallai, I., Gazit, D., Boyce, M.C., Ortiz, C., 2012. Three-dimensional structure of the shell plate assembly of the chiton Tonicella marmorea and its biomechanical consequences. Journal of Structural Biology, 177, 314-328.

Daget, J., Gayet, M., Meunier, F.J., Sire, J.-Y., 2001. Major discoveries on the dermal skeleton of fossil and recent polypteriforms: a review. Fish and Fisheries. 1, 113-124.

Dryden, I.L. \& Mardia, K.V. (eds.), 1998. Statistical Shape Analysis, Wiley, West Sussex.

Dunlop, J.W.C., Weinkamer, R., Fratzl, P., 2011. Artful interfaces within biological materials. Materials Today, $14,70-78$.

Duro-Royo J., Zolotovsky K., Mogas-Soldevila L., Varshney S., Oxman N., Boyce M.C., Ortiz C., 2015. MetaMesh: A hierarchical computational model for design and fabrication of biomimetic armor surfaces. Computer-Aided Design, 16, 14-27.

Elewa, A.M.T. (ed.), 2010. Morphometrics for Nonmorphometricians. Springer, New York.

Gemballa S., Bartsch P., 2002. Architecture of the integument in lower Teleostomes: Functional morphology and evolutionary implications. Journal of Morphology, 253, 290-309.

Gueziec, E., 1996. Spline curves and surfaces for data modelling. In: Marcus, L., Corti, M., Loy, A., Naylor, G. J. P., Slice, D.E. (eds.), Advances in Morphometrics. Plenum Press, New York. Han, L., Wang, L, Song, J., Boyce, M. C., Ortiz, C., 2011. Direct quantification of the mechanical anisotropy and fracture of an individual exoskeleton layer via uniaxial compression of micropillars. Nano Letters, 11, 3868-3874. 
Humphries, J., 2003. Polypterus senegalus, (On-line), Digital Morphology. Accessed January 12, 2015 at http://digimorph.org/specimens/Polypterus_senegalus/whole/.

Kendall, D.G., 1977. The diffusion of shape. Advances in Applied Probability, 9, 428-430.

Krauss, S., Monsonego-Ornan, E., Zelzer, E., Fratzl, P., Shahar, R., 2009. Mechanical function of a complex three-dimensional suture joining the bony elements in the shell of the red-eared slider turtle. Advanced Materials, 21, 407-412.

Li, Y., Ortiz, C., Boyce, M. C., 2011. Stiffness and strength of suture joints in nature. Physical Review E, 84, 062904, 1-5.

Li, Y., Ortiz, C., Boyce, M. C., 2012. Bioinspired, mechanical, deterministic fractal model for hierarchical suture joints. Physical Review E, 85, 031901, 1-14.

Li, L., Ortiz, C., 2013. Biological design for simultaneous Optical transparency and mechanical robustness in the shell of Placuna placenta. Advanced Materials, 25, 2344-2350.

Little, J., Mardia, K., 1996. Edgels and tangent planes in image warping. In: Marcus, L., Corti, M., Loy, A., Naylor, G. J. P., Slice, D.E. (eds.), Advances in Morphometrics. Plenum Press, New York. Meinke, D.K., Skinner, H.C.W., Thomson, K.S., 1979. X-ray diffraction of the calcified tissues in Polypterus. Calcified Tissue International, 28, 37-42.

Meunier, F.J., 1987. Os cellulaire, os acellulaire et tissus derives chez les Osteichthyens: Les phonomenes de l'acellularisation et de la perte de mineralisation. Annals of Applied Biology, 4, 201-225.

Meyers, M. A., Chen, P. Y., Lin, A. Y. M., Seki, Y., 2008. Biological materials: structure and mechanical properties. Progress in Materials Science, 53, 1-206.

Ortiz, C., Boyce, M. C., 2008. Bioinspired structural materials. Science, 319, 1053-1054. 
Oyen, M. L., Bushby, A. J., Mann, A., Ortiz, C., 2006. Mechanics of biological and biomimetic materials at small length scales. Journal of Materials Research, 21, 1869-1870.

Pearson, D.M., 1981. Functional aspects of the integument in polypterid fishes. Zoological Journal of the Linnean Society, 72, 93-106.

Raabe, D., Sachs, C., Romano, P., 2005. The crustacean exoskeleton as an example of a structurally and mechanically graded biological nanocomposite material. Acta Materialia, 53, 4281-4292.

Sire, J.-Y., 1989. Scales in young Polypterus senegalus are elasmoid: New phylogenetic implications. The American Journal of Anatomy, 186, 315-323.

Sire J.-Y., 1990. From ganoid to elasmoid scales in the Actinopterygian fishes. Netherlands Journal of Zoology, 40, 75-92.

Sire, J.-Y., 1994. Light and TEM study of nongenerated and experimentally regenerated scales of Lepisoteus oculatus (Holostei) with particular attention to ganoine formation. Anatomical Record, 240, $189-207$.

Song., J., Reichert, S., Kallai, I., Wund, M., Boyce, M.C., Ortiz, C., 2010. Quantitative microstructural studies of the armor of the marine threespine stickleback (Gasterosteus aculeatus). Journal of Structural Biology, 171, 318-331.

Song, J., Ortiz, C., Boyce, M. C., 2011. Threat protection mechanics of an armored fish. Journal of the Mechanical Behavior of Biomedical Materials, 4, 699-712.

Tai, K., Dao, M., Suresh, S., Palazoglu, A., Ortiz, C., 2007. Nanoscale heterogeneity promotes energy dissipation in bone. Nature Materials, 6, 454-462.

Tytell, E.D., Lauder, G.V., 2002. The C-start escape response of Polypterus senegalus: bilateral muscle activity and variation during stage 1 and 2. Journal of Experimental Biology, 205, 2591-2603. 
Wainwright, S. A., Biggs, W. D., Currey, J. D., Gosline, J. M., 1976. Mechanical Design in Organisms, Princeton University Press, Princeton.

Wang, L.F., Song, J.H., Ortiz, C., Boyce, M.C., 2009. Anisotropic design of a multilayered biological exoskeleton. Journal of Materials Research, 24, 3477-3494.

Weber, G.W. (ed.), 2011. Virtual Anthropology: A guide to a new interdisciplinary field. Springer, New York.

Weber G.W., Bookstein, F.L., Strait, D.S., 2011. Virtual anthropology meets biomechanics. Journal of Biomechanics, 44, 1429-1432.

Weiner, S., Addadi, L, 1997. Design strategies in mineralized biological materials. Journal of Materials Chemistry, 7, 689-702.

Wyneken, J., Godfrey, M. H., Bels, V. (Eds.), 2008. Biology of turtles: From structures to strategies of life. CRC Press, Boca Raton.

Yang, W., Chen, I.H., Gluduvatz, B., Zimmermann, E.A., Ritchie, R.O., Meyers, M.A., 2013. Natural flexible dermal armor. Advanced Materials, 25, 31-48.

Yourdkhani, M., Pasini, D., Barthelat, F., 2011. Multiscale mechanics and optimization of gastropod shells. Journal of Bionic Engineering, 8, 357-368.

Zelditch, M.L., Swiderski, D.L., Sheets, D.H. \& Fink, W.L. (Eds.). 2004. Geometric Morphometrics for Biologists: A Primer. Elsevier Academic Press, New York. 


\begin{tabular}{ccl}
\hline Landmark & Type & Description \\
\hline 1 & I & Posterior corner \\
2 & III & Midpoint between dorsal and posterior corners \\
3 & I & Dorsal corner \\
4 & II & Dorsal base of peg \\
5 & III & Midpoint between dorsal base and tip of peg \\
6 & I & Tip of peg \\
7 & III & Midpoint between anterior base and tip of peg \\
8 & II & Anterior base of peg \\
9 & II & Anterior point of anterior margin transition (interserial overlap) \\
10 & I & Tip of anterior process (most anterior point) \\
11 & II & Anterior point of paraserial overlap transition \\
12 & II & Anterior margin valley \\
13 & III & Midpoint between anterior margin valley and anterior margin peak \\
14 & II & Anterior margin peak \\
15 & II & Ventral point of paraserial overlap transition \\
16 & I & Posterior point of anterior margin transition (interserial overlap) \\
17 & I & Ventral corner \\
18 & I & Midpoint of socket on edge of scale \\
19 & II & Point between tip of peg and socket on the top side of scale \\
20 & II & Central base of peg \\
\hline & & \\
\hline
\end{tabular}




\begin{tabular}{lll}
\hline Parameter & Description & Calculation \\
\hline PL & Peg length & Distance between LM6-LM20 \\
APL & Anterior process length & Distance between LM10-LM12 \\
DAR & Diagonal aspect ratio & Distance between LM3-LM16 / Distance between LM1-LM10 \\
$\gamma$ & Peg tip angle & Angle between LM4-LM6-LM8 \\
$\theta$ & Anterior process angle & Angle between LM10-LM19-LM6 \\
V & Volume of scale & Volume of the STL object of the scale \\
$\mathrm{U}$ & Strain energy & $\mathrm{U}=\sum_{m, q} u(m, q) * t^{2}$, where $u(m, q)=\frac{1}{2}\left[(2 \mu+\lambda) *\left(\varepsilon_{11}(\mathrm{~m}, \mathrm{q})^{2}+\right.\right.$ \\
& & $\left.\left.\varepsilon_{22}(\mathrm{~m}, \mathrm{q})^{2}\right)+2 \lambda *\left(\varepsilon_{11}(\mathrm{~m}, \mathrm{q})^{*} \varepsilon_{22}(\mathrm{~m}, \mathrm{q})\right)+\mu \gamma_{12}(\mathrm{~m}, \mathrm{q})^{2}\right]$ \\
\hline
\end{tabular}




\begin{tabular}{|c|c|c|c|c|c|}
\hline & & & & \\
\hline & & Anterior & Tail & Ventral & Fin \\
\hline \multirow{7}{*}{$\begin{array}{l}\text { Average } \\
(\mathrm{N}=30)\end{array}$} & PL & $*$ & $*$ & $*$ & $*$ \\
\hline & APL & 0.90 & * & $*$ & $*$ \\
\hline & DAR & $*$ & $*$ & $*$ & $*$ \\
\hline & $\gamma$ & 0.24 & $*$ & $*$ & * \\
\hline & $\theta$ & 0.87 & $*$ & $*$ & * \\
\hline & V & $*$ & $*$ & $*$ & $*$ \\
\hline & $\mathrm{U}$ & 0.21 & $*$ & $*$ & $*$ \\
\hline \multirow{7}{*}{$\begin{array}{c}\text { Anterior } \\
(\mathrm{N}=36)\end{array}$} & $\mathrm{PL}$ & & $*$ & $*$ & $*$ \\
\hline & APL & & $*$ & $*$ & * \\
\hline & DAR & & $*$ & $*$ & $*$ \\
\hline & $\gamma$ & & $*$ & $*$ & * \\
\hline & $\theta$ & & $*$ & $*$ & $*$ \\
\hline & V & & $*$ & $*$ & $*$ \\
\hline & $\mathrm{U}$ & & * & $*$ & $*$ \\
\hline \multirow{7}{*}{$\begin{array}{c}\text { Tail } \\
(\mathrm{N}=21)\end{array}$} & PL & & & $*$ & 0.84 \\
\hline & APL & & & $*$ & 0.91 \\
\hline & DAR & & & $*$ & $*$ \\
\hline & $\gamma$ & & & $*$ & $*$ \\
\hline & $\theta$ & & & 0.06 & $*$ \\
\hline & V & & & 0.80 & * \\
\hline & $\mathrm{U}$ & & & 0.11 & $*$ \\
\hline \multirow{7}{*}{$\begin{array}{c}\text { Ventral } \\
(\mathrm{N}=41)\end{array}$} & $\overline{P L}$ & & & & * \\
\hline & APL & & & & $*$ \\
\hline & DAR & & & & $*$ \\
\hline & $\gamma$ & & & & * \\
\hline & $\theta$ & & & & * \\
\hline & V & & & & $*$ \\
\hline & $\mathrm{U}$ & & & & $*$ \\
\hline
\end{tabular}

